\title{
MLL1 and DOT1L cooperate with meningioma-1 to induce acute myeloid leukemia
}

\author{
Simone S. Riedel, ${ }^{1}$ Jessica N. Haladyna, ${ }^{1}$ Matthew Bezzant, ${ }^{1}$ Brett Stevens, ${ }^{2}$ Daniel A. Pollyea, ${ }^{2}$ Amit U. Sinha, ${ }^{3}$ \\ Scott A. Armstrong, ${ }^{3}$ Qi Wei, ${ }^{4}$ Roy M. Pollock, ${ }^{5}$ Scott R. Daigle, ${ }^{5}$ Craig T. Jordan, ${ }^{2}$ Patricia Ernst, ${ }^{1}$ Tobias Neff, ${ }^{1}$ and Kathrin M. Bernt ${ }^{1}$ \\ 'Division of Pediatric Hematology/Oncology/BMT, University of Colorado School of Medicine and Children's Hospital Colorado, Aurora, Colorado, USA. ²ivision of Hematology, University of Colorado Denver, \\ Aurora, Colorado, USA. 'Bivision of Hematology/Oncology, Children's Hospital, and Department of Pediatric Oncology, Dana-Farber Cancer Institute, Harvard Medical School, Boston, Massachusetts, USA. \\ ${ }^{4}$ Department of Pathology, Children's Hospital Colorado, Aurora, Colorado, USA. ${ }^{5}$ Epizyme Inc., Cambridge, Massachusetts, USA.
}

\begin{abstract}
Meningioma-1 (MN1) overexpression is frequently observed in patients with acute myeloid leukemia (AML) and is predictive of poor prognosis. In murine models, forced expression of MN1 in hematopoietic progenitors induces an aggressive myeloid leukemia that is strictly dependent on a defined gene expression program in the cell of origin, which includes the homeobox genes Hoxa9 and Meis1 as key components. Here, we have shown that this program is controlled by two histone methyltransferases, MLL1 and DOT1L, as deletion of either MI11 or Dot11 in MN1-expressing cells abrogated the cell of originderived gene expression program, including the expression of Hoxa cluster genes. In murine models, genetic inactivation of either MII1 or Dot1I impaired MN1-mediated leukemogenesis. We determined that HOXA9 and MEIS1 are coexpressed with MN1 in a subset of clinical $M N 1^{h i}$ leukemia, and human $M N 1^{\text {hi }} / H O X A 9^{\text {hi }}$ leukemias were sensitive to pharmacologic inhibition of DOT1L. Together, these data point to DOT1L as a potential therapeutic target in MN1 ${ }^{\text {hi }} \mathrm{AML}$. In addition, our findings suggest that epigenetic modulation of the interplay between an oncogenic lesion and its cooperating developmental program has therapeutic potential in AML.
\end{abstract}

\section{Introduction}

The meningioma-1 (MN1) gene is frequently overexpressed in acute myeloid leukemia (AML) and is associated with a poor prognosis (1-5). High MN1 expression occurs across multiple cytogenetic and molecular subgroups of AML (4-6). Two distinct subtypes of AML are negatively associated with high MN1 expression levels: AML with mutations in nucleophosmin 1 (NPM1c) (1-5) and AML with a translocation of the mixed lineage leukemia gene, $M L L$ $(4,6)$. The highest expression levels of MN1 have been reported in patients with an inversion of chromosome 16 (inv[16]), and 100\% of inv(16) AML overexpress MN1 $(4,6)$. In apparent contradiction to the poor outcome associated with high MN1 expression, inv(16) AML has a favorable prognosis. However, inv(16) AML represents only a small subgroup of $M N 1^{h i}$ AML. A second subgroup associated with higher-than-average MN1 expression levels is AML with a complex karyotype (4). Outcomes for AML with a complex karyotype, as well as $M N 1^{h i}$ AML as a whole, are poor.

MN1 was first described as part of a translocation in meningioma (7). MN1 is a transcriptional coactivator that cooperates with

Authorship note: S.S. Riedel and J.N. Haladyna, and T. Neff and K.M. Bernt, contributed equally to this work.

Conflict of interest: A.U. Sinha is the founder of Basepair. S.A. Armstrong is a consultant for Epizyme Inc. R.M. Pollock is a former employee of Epizyme Inc. S.R. Daigle is a current employee of Epizyme Inc. P. Ernst holds Amgen stock; this is in no relation to this project. T. Neff is a coinventor on US Patent 62/026583 (Dot1l Inhibition in Patients with MN1 High AML) filed 7/2014. K.M. Bernt is a coinventor on US Patent 62/026583 (Dot1l Inhibition in Patients with MN1 High AML) filed 7/2014.

Submitted: January 12, 2015; Accepted: January 14, 2016.

Reference information: / Clin Invest. 2016;126(4):1438-1450. doi:10.1172/JCI80825. the nuclear receptors for retinoic acid (RAR) (8) and vitamin D (9), possibly through direct binding of other coactivators such as RAC3 and p300/CBP (8). In AML, MN1 is frequently overexpressed and occasionally fused to TEL as part of the rare MN1-TEL translocation (10). Exactly how MN1 contributes to leukemogenesis is still not fully understood. A dominant negative effect on RAR/TEL signaling has been described for the MN1-TEL fusion (11), but this mechanism may not apply to MN1 overexpression. Importantly, MN1 has little structural similarity to any other protein $(12,13)$. Pharmacologic targeting of high MN1 expression in AML has so far remained elusive.

MN1 overexpression in murine hematopoietic progenitors induces a rapidly fatal myeloid leukemia, reflecting the strong transforming ability of MN1 (6, 14-16). Forced expression of MN1 induces proliferation and a block in differentiation, mapped to the $\mathrm{N}$ - and C-terminus of MN1, respectively $(13,16)$. The developmental window, during which MN1 is able to induce leukemia, is narrow and well defined; neither purified hematopoietic stem cells (HSCs) nor cells beyond the common myeloid progenitor (CMP) can serve as the cell of origin (14). Heuser et al. pinpointed the transcriptional requirements for the cell of origin in the MN1 leukemia mouse model. Progenitors that can serve as a target cell for MN1-mediated transformation are characterized by a distinct gene expression profile that is shut down at the transition from CMP to granulocyte macrophage progenitor (GMP). Key components of this program include the Hoxa cluster genes Hoxa9 and Hoxa10, as well as Meis1. Forced expression of Hoxa9/10 and Meis1 rendered GMPs as well as HSCs susceptible to MN1-mediated transformation. This suggests that MN1 alone is unable to induce the full progenitor program (including the later Hoxa cluster) 
A

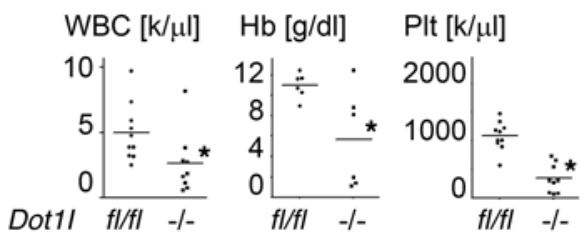

C

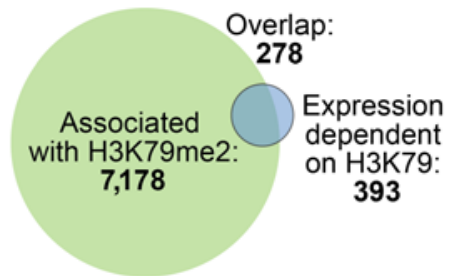

D

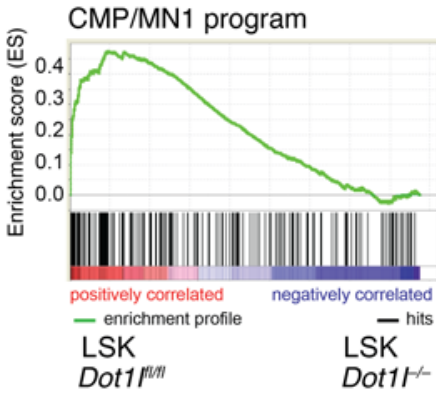

LSK

Dot1 $1^{-}$
B Dot11 Dot11

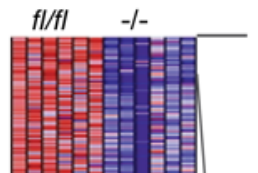

NES $=2.6$

$P<0.001$
Top 30

\section{Bcl11a}

Pbx1

Tnfrsf13c

Rab27a

Calcoco1

Jkamp

HIf

Rnf169

4930520004Rik

Bcl11a

Hemgn

KIf3

Taf5I

Trp53inp1

Mtap4

Flt3

Lasp1

Nanp

Armcx1

Hoxas

Mapkbp1

Zfp608

Aldh6a1

II12a

Gcnt2

Tspan13

Nceh1

Traf6

Phactr4

Bcl11a

422: Meis1 $(P<0.05)$
Figure 1. The MN1 cooperating program is enriched in Dot1/ WT versus KO LSK cells. (A) Total WBC, hemoglobin ( $\mathrm{Hb})$, and platelet (PIt) count in Dot $\left.1\right|^{\mathrm{fl} / \mathrm{fl}}(\mathrm{fl} / \mathrm{fl}, n=$ 10) and $M \times 1-\left.C r e \operatorname{Dot}_{1}\right|^{f / f \mid}(-/-, n=9)$ mice 3 weeks after the induction of $\mathrm{Cre}$. Two-sided $t$ test $\operatorname{Dot} 11^{-/-}$vs. Dot $1 f^{|f / f|} ;{ }^{*} P<0.05$. (B) Heatmap of expression array data of sorted LSK cells from Dot $11^{f / / f l}(\mathrm{fl} / \mathrm{fl}$ ) and Mx1-Cre $\operatorname{Dot}_{1 / f / f l}(-/-)$ mice 12 days after induction of Cre. Shown are all probe sets/genes with differential expression at $P<0.05$ (393 genes), as well as a list of the top 30 differentially expressed probe sets and Meis1; $n=$ 6 mice per group. (C) Venn diagram of genes associated with $\mathrm{H} 3 \mathrm{~K} 79$ dimethylation in LSK cells by ChIP-Seq (18) and genes downregulated after loss of Dot1/ in LSK cells. (D) GSEA showing enrichment of the CMP/MN1 program in Dot $1 f^{f / f f}$ vs. Dot 1//- LSK cells. NES and $P$ value according to ref. 43 . required for transformation. However, expression of Hoxa9/10 and Meis1 remains high in MN1-AML cells with phenotypic and morphologic characteristics beyond the CMP stage; thus, MN1 may prevent the developmentally appropriate shutdown of these important loci at the CMP-to-GMP transition. Despite the ability to induce leukemia as a single hit in murine CMPs, forced expression of MN1 failed to fully transform human umbilical cord blood cells. However, cotransduction of MN1 and NUP98-HOXD13 resulted in full transformation to AML. This confirms the central role of homeobox transcription factors and their collaboration with MN1 in AML leukemogenesis (17).

The later Hoxa cluster genes and Meis1 are also critical direct-binding targets of fusions of the mixed lineage leukemia gene, MLL1. MLL rearrangements occur in both myeloid malignancies and acute lymphoblastic leukemia (ALL) and are associated with a poor prognosis, particularly in infant ALL. We recently showed that the histone methyltransferase DOT1L is absolutely required in MLL-AF9 leukemia and that the mechanism involves a specific dependence of MLL fusion target gene expression on functional DOT1L (18). Based on these data, a small molecule inhibitor of DOT1L is currently in phase I/II clinical development for $M L L$-rearranged leukemia $(19,20)$. DOT1L methylates histone 3 on lysine 79 (H3K79); it is the predominant H3K79 methyltransferase and responsible for monomethylation (me1), dimethylation (me2), and trimethylation (me3) of H3K79 (21). H3K79 methylation is present on MLL fusion target genes in $M L L$-rearranged leukemia but also on the later Hoxa cluster genes and Meisl in normal hematopoietic progenitors at the developmental stage where these genes are highly expressed (18). The later Hoxa cluster and Meis1 are also regulated in a DOT1L-dependent manner in non-MLL-rearranged CALM-AF1O (22) and NUP98-NSD1 (23) fusion-driven leukemia.
Given the poor outcome associated with high MN1 expression and the ability of MN1 to act as a strong oncogenic driver, MN1 could represent an attractive target for therapeutic intervention. However, no small molecule inhibitor for MN1 has been described so far. Based on the dependence of MN1-driven leukemia on HOXA9 expression, we hypothesized that leukemias driven by MN1 are sensitive to DOT1L inhibition via downregulation of the cooperating later HOXA cluster genes. This would suggest that DOT1L could be a therapeutic target in $M N 1^{h i} \mathrm{AML}$.

\section{Results}

Loss of Dot1l in normal early hematopoietic progenitors leads to downregulation of a distinct gene expression program. We aimed to delineate early gene expression changes that occur after genetic inactivation of Dot1l in normal hematopoiesis. To this end, we crossed conditional Dot $11^{l / f l}$ mice into the $\mathrm{M} \times 1$-Cre model, which allows rapid and precise excision of exon 5 of the Dot1l gene (which contains most of the active site) after 3 doses of polyinosinic:polycytodylic acid (pI:pC) (Supplemental Figure 1, A and B; supplemental material available online with this article; doi:10.1172/ JCI80825DS1). Induced Dot1 $1^{\text {fl/ }} \mathrm{M}$ M1-Cre mice developed pancytopenia similar to data previously reported for conditional Dot1l inactivation models using tamoxifen-inducible systems (Figure 1A and refs. 24, 25). In the Mx1-Cre model, loss of functional Dot1l was confined to the hematopoietic system, and the high efficiency of the Mx1-Cre promoter allowed analysis of cell-autonomous gene expression changes at a defined early time point. We isolated Lin $^{-} \mathrm{Sca}-1^{+} \mathrm{c}-\mathrm{Kit}^{+}(\mathrm{LSK})$ cells 6 days after pI:pC injection. The interferon response elicited by pI:pC treatment has been shown to lead to a temporary loss of quiescence in the HSC compartment and distorts the ability to isolate $\mathrm{HSC}$ /progenitors using flowcytomet- 
A ${ }^{\mathrm{MN} 1 \mathrm{CMP}-\mathrm{T}}$

Colony number

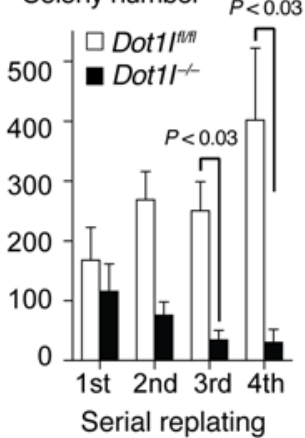

Cell number (k/plate)

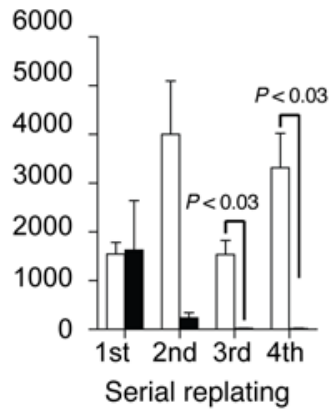

B Colony number
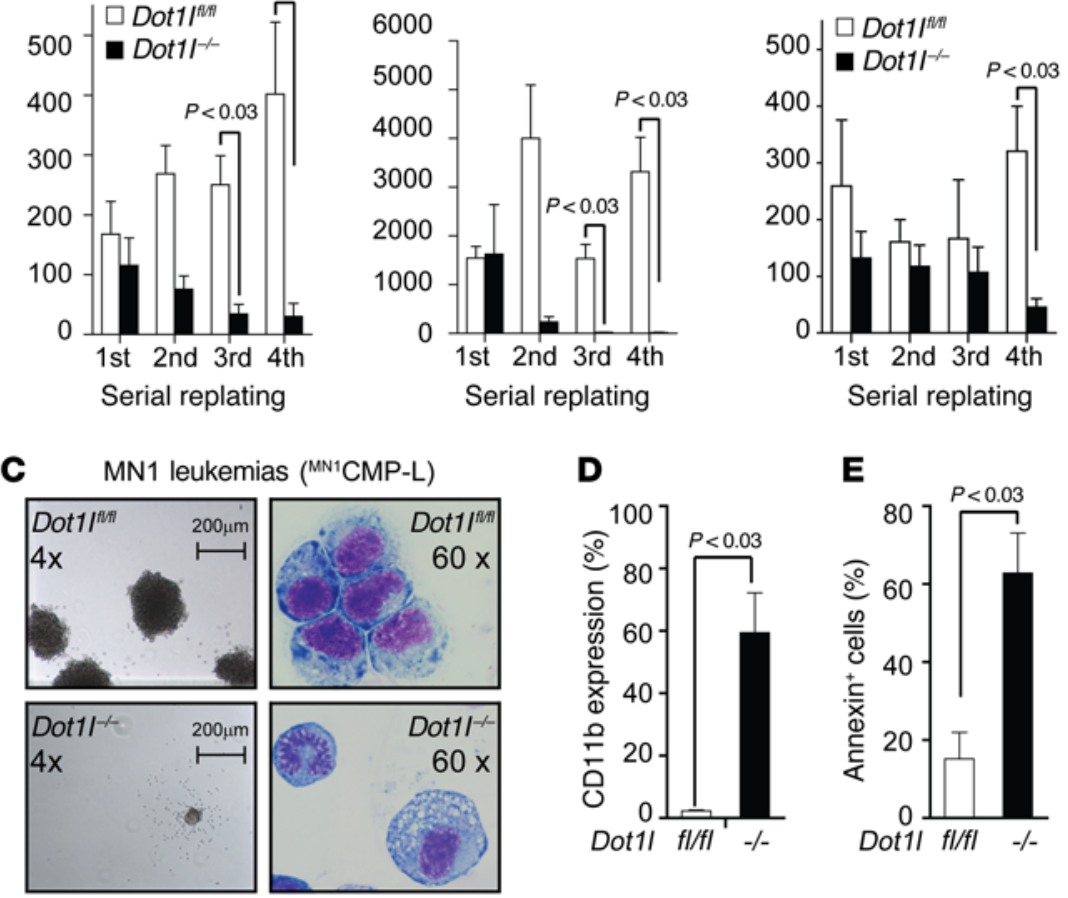

D

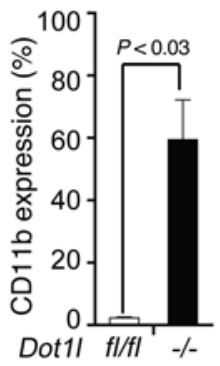

E

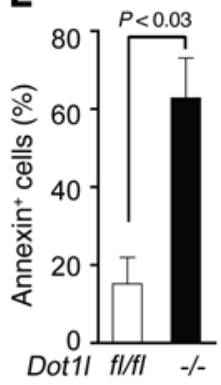

Cell number (k/plate)

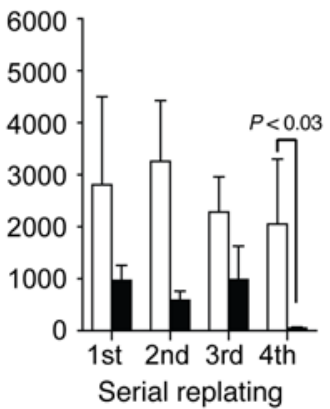

$\mathbf{F}$

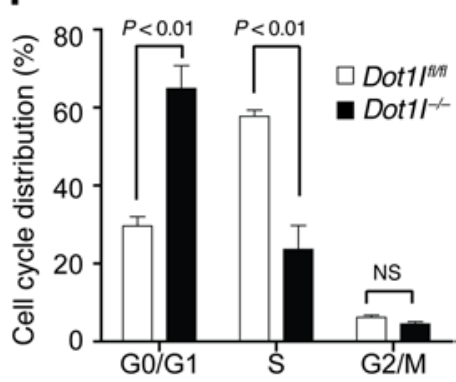

Figure 2. Loss of Dot1l leads to decreased growth, increased differentiation, and apoptosis of MN1-driven CMP-derived murine leukemia cells. (A) Serial replating of MN1-transformed CMPs ( $\left.{ }^{\mathrm{MN} 1} \mathrm{CMP}-\mathrm{T}\right)$ after Cre-induced loss of Dot1l. Left plot: number of colonies per 500 plated cells; right plot: total cell number. $n=5$ independent experiments; two-sided $t$ test Dot $11^{-/-}$vs. Dot $11^{f / f l}$. Error bars represent \pm SEM. (B) Serial replating of MN1-driven murine leukemias (MN1CMP-L) after Cre-induced loss of Dot1l. Left plot: number of colonies per 500 plated cells; right plot: total cell number. Dot1/f/fl: bulk population from 3 independent experiments; Dot1//-: 2 bulk populations and 4 individually picked clones (due to outgrowth of nondeleted cells) from 3 independent experiments. Two-sided $t$ test Dot1//- vs. Dot $1 f^{f / f l}$. Error bars represent \pm SEM. (C) Methylcellulose colony and leukemia cell morphology (Wright-Giemsa stain) of MN1-driven murine leukemias ( ${ }^{M N 1}$ CMP-L) 14 days after transduction with Cre. Representative of 3 independent expermients. (D) CD11b expression in MN1driven murine leukemias ( $\left.{ }^{\mathrm{MN}} \mathrm{C} \mathrm{CMP}-\mathrm{L}\right) 3$ weeks after deletion of Dot11. Dot $1 /^{f / f l}$ : bulk population from 3 independent experiments; Dot1//-- 2 bulk populations and 4 individually picked clones (due to outgrowth of nondeleted cells) from 3 independent experiments. Two-sided $t$ test Dot $11^{-/-}$vs. Dot $11^{\prime / / f I}$. Error bars represent \pm SEM. (E) Apoptosis (Annexin staining) in MN1-driven murine leukemias ( ${ }^{\text {MN1 }} \mathrm{CMP}-\mathrm{L}$ ) 3 weeks after deletion of Dot1/. Dot1/f/fl: bulk population from 3 independent experiments; Dot1//- 2 bulk population and 4 individually picked clones (due to outgrowth of nondeleted cells) from 3 independent experiments. Two-sided $t$ test Dot1/ ${ }^{-/-}$vs. Dot1/fl/fl. Error bars represent \pm SEM. (F) Cell cycle distribution (EdU incorporation/DAPI staining) in MN1-driven murine leukemias ( $\left.{ }^{\mathrm{MN}} \mathrm{CMP}-\mathrm{L}\right) 3$ weeks after deletion of Dot1l. Dot $1 /^{\mathrm{fl} / \mathrm{fl}}$ : bulk population from 3 independent experiments; Dot $11^{-/-}: 2$ bulk population and 4 individually picked clones (due to outgrowth of nondeleted cells) from 3 independent experiments. Two-sided $t$ test Dot $11^{-/-}$vs. Dot $1 f^{f / f l}$. Error bars represent \pm SEM.

ric markers. However, these effects were resolved after 5 days (26), and flowcytometric analysis of $\mathrm{pI}: \mathrm{pC}$-injected animals performed 6 days after the last dose showed a clearly distinguishable LSK population (Supplemental Figure 1C). We performed gene expression analyses comparing pI:pC-injected Dot $1^{f / f l}$ Mx1-Cre LSK cells $\left(\operatorname{Dot} 1 l^{-/}\right)$to LSK cells from pI:pC-injected Dot $1 l^{f / f l}$ littermates $\left(\operatorname{Dot} 1 f^{f / f l}\right)$. Similar to results previously reported in $M L L$-rearranged leukemias, loss of Dot1l led to downregulation of a specific set of genes without inducing global transcriptional changes (463 probes, corresponding to 393 genes at $P<0.05$; Figure $1, \mathrm{~B}$ and C, and Supplemental Table 1). We defined the set of genes with decreased expression in Dot1l ${ }^{-/}$LSK cells as "DOT1L-dependent in LSK" (Supplemental Table 2). As expected, the majority of the genes that were dependent on DOT1L in LSK cells were associated with high levels of H3K79 dimethylation downstream of the transcription start site (as determined by ChIP sequencing [ChIP-Seq] in ref. 18 and Figure 1C). Gene set enrichment analysis indicated that this gene set has significant overlap with genes regulated by
DOT1L in $M L L$-rearranged leukemias, including the key MLLfusion downstream target genes Hoxa9 and Meis1 (Supplemental Figure 1D). In addition, many of these genes are downregulated at the LSK-to-GMP transition (Supplemental Figure 1E).

The MN1 leukemogenic gene expression program is dependent on functional DOT1L in LSK cells. Heuser et al. (14) reported that a specific gene expression program in CMPs overlapped with the gene expression program defining MN1 leukemias, and key components of this CMP program cooperated with MN1 to cause myeloid leukemia. We asked whether the MN1 gene expression program (i.e., high in CMP vs. GMP, and MN1 vs. GR1/CD11b+ in ref. 14, "CMP/MN1 program"), is dependent on DOT1L in normal early hematopoietic progenitors. Indeed, gene set enrichment analysis (GSEA) demonstrated a strong enrichment of the $\mathrm{CMP} / \mathrm{MN} 1$ program gene set in the DOT1L-dependent gene set in normal LSK cells (increased in Dot $1 b^{f l / f l}$ vs. Dot $1 l^{-/}$) (Figure 1D), as well as in the DOT1L-dependent genes in MLL-AF9 leukemias (Supplemental Figure 1F). 
A Primary Recipients

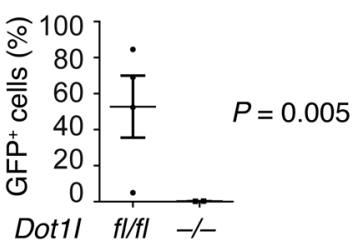

\section{Secondary Recipients}

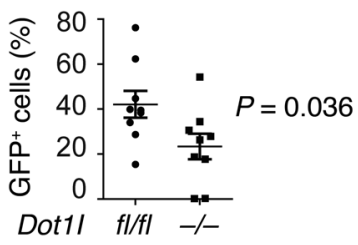

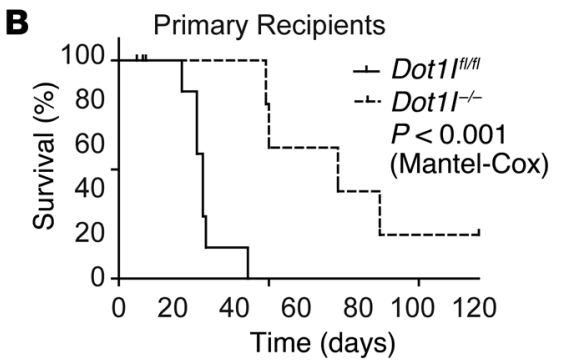

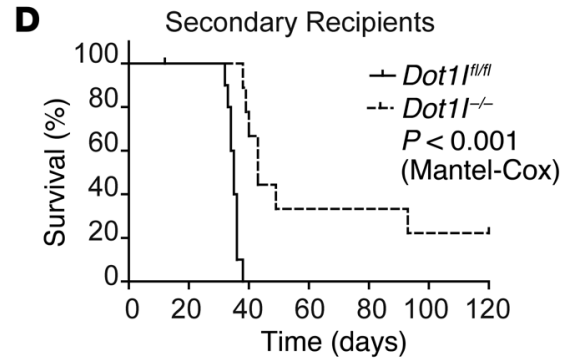

Figure 3. CMP-derived murine MN1-driven leukemia initiation and maintenance are dependent on functional DOT1L in vivo. (A) Leukemic burden (\% of GFP+ cells in the peripheral blood) in recipients on day 20 after injection of MN1 in vitro transformed

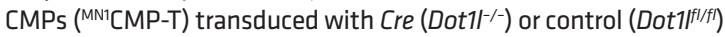

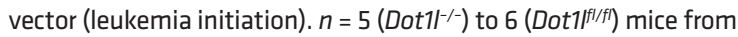
2 individual experiments. Two-sided $t$ test Dot11/-- vs. Dot $1 f^{f / f f l}$. Error bars represent \pm SEM. (B) Survival of recipients of MN1 in vitro transformed CMPs ( $\left.{ }^{\left({ }^{N 1}\right.} \mathrm{CMP}-\mathrm{T}\right)$ transduced with Cre $\left(\mathrm{Dot}_{1 /-1}\right)$ or control (Dot7/f/ff) vector (leukemia initiation). $n=6$ (Dot11 $1^{-1-}$ ) to 7 (Dot $\left.1 f^{f / f f}\right)$ mice from 2 individual experiments (Mantel-Cox). (C) Leukemic burden (\% of GFP+ cells in the peripheral blood) in recipients on day 20 after injection of MN1-driven CMP-derived leukemias (MN1CMP-L) transduced with Cre (Dot11/-) or control $\left(D o t f^{f / f f}\right)$ vector (leukemia maintenance). $n=9$ mice per group from 2 individual experiments. Two-sided $t$ test Dot $11^{-1-}$ vs. $\operatorname{Dot}^{\mid f / f f}$. Error bars represent \pm SEM. (D) Survival of recipients of MN1-driven CMP-derived leukemias ( ${ }^{\mathrm{MN}} \mathrm{CMP}-\mathrm{L}$ ) transduced with $\mathrm{Cre}\left(\operatorname{Dot} 11^{-/-}\right)$or control $\left(\left.\operatorname{Dot}\right|^{(f / / f)}\right)$ vector (leukemia maintenance). $n=9\left(\operatorname{Dot} 11^{-/-}\right)$to $11\left(\operatorname{Dot} 11^{f / f f}\right)$ mice from 2 individual experiments (Mantel-Cox).
MN1-induced CMP-derived $A M L$ is dependent on functional Dot1l. Hoxa9/Meis1 expression in the cell of origin is critically important for the ability of MN1 to induce AML (14). Based on our findings showing that Hoxa9, Hoxa10, and Meis1 expression are dependent on functional DOT1L in early hematopoietic progenitors, we asked whether this dependency on DOT1L was preserved in MN1 leukemias. We introduced the human MN1 cDNA into sorted Dot $1 l^{t / f l}$ CMPs to establish in vitro transformed ${ }^{\mathrm{MN} 1} \mathrm{CMP}-\mathrm{T}$. Deletion of Dot $1 l$ through introduction of Cre (Dot $\left.1 l^{-/ \mathrm{MN} 1} \mathrm{CMP}-\mathrm{T}\right)$ resulted in reduced cell numbers and colonies in serial replating assays (Figure 2A). We also injected MN1-transduced Dot $1 b^{t / / f l}$ CMPs into recipient mice to establish Dot1l-conditional leukemias ( $\left.{ }^{\mathrm{MN} 1} \mathrm{CMP}-\mathrm{L}\right)$. Excision of exon 5 of Dot 1 l in ${ }^{\mathrm{MN} 1} \mathrm{CMP}-\mathrm{L}$ isolated from moribund mice again resulted in decreased replating efficiency and decreased cell numbers (Figure 2B). Dot $1 l^{-/-}$colonies

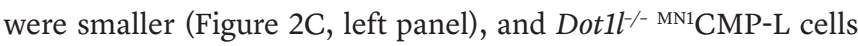
showed morphologic signs consistent with increased differentiation (Figure 2C, right panel). This was reflected in an increase in the expression of the myeloid differentiation marker CD11b

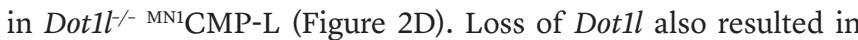
increased apoptosis (Figure 2E) and a decrease in the fraction of cycling cells (Figure 2F). Similar results were observed in Dot $11^{-/-}$ ${ }^{\mathrm{MN} 1} \mathrm{CMP}-\mathrm{T}$ cells (Supplemental Figure 2, A-C). While the smaller colony size and increased differentiation mimic the effect of loss of Dot 1 l in $M L L$-rearranged leukemias, we observed several subtle differences between the two models. Loss of Dot1l in $M L L$-rearranged leukemias causes a minimal increase in apoptosis, while apoptosis in $\mathrm{Dot}_{1 l^{-/}} \mathrm{MN} 1$ leukemias was more pronounced. More importantly, while we were unable to isolate viable and proliferating Dot $11^{-/}$MLL-AF9 cells beyond the third replating, serial replating of Dot $1 l^{-/-}$MN1 leukemias was inefficient but possible.

We next analyzed the effect of loss of functional DOT1L on in vivo MN1-leukemias. Dot $1 l^{t / f l}{ }^{\mathrm{MN}} \mathrm{CMP}-\mathrm{T}$ were transduced with Cre (or control) and injected into primary recipients to interrogate leukemia initiation. To assess the effect of loss of DOT1L in leukemia maintenance, $\operatorname{Dot} 1 t^{t / f l}{ }^{\mathrm{MN} 1} \mathrm{CMP}-\mathrm{L}$ were isolated from moribund mice, transduced with Cre (or control), and transplanted into secondary recipients. While control mice succumbed to leukemia within 3-4 weeks, Dot1t/- MN1CMP-T- or ${ }^{\mathrm{MN} 1} \mathrm{CMP}-\mathrm{L}-$ injected animals experienced significantly decreased leukemic burden (Figure 3, A and C) and prolonged survival (Figure 3, B and D). All leukemias that eventually did develop in Cre-transduced cohorts were found to contain at least one floxed (unexcised) Dot 1 l allele by genotyping (Supplemental Figure 3) and had thus escaped full genetic inactivation of Dot1l.

Loss of DOT1L leads to downregulation of the MN1-cooperating program in MN1-transformed CMPs. We next determined the H3K79 methylation status of the MN1-bound genes using ChIPSeq for H3K79me2 in MN1-driven leukemias. Indeed, high levels of H3K79 dimethylation were observed close to the transcription start site of the gene set that was previously published to be bound by MN1 (ref. 14, Figure 4, A and B, and Supplemental Table 2). We next asked whether loss of functional Dot1l in CMP-derived MN1-transformed cells resulted in downregulation of the CMP/ MN1 program, including the key loci Hoxa9 and Meis1. We performed quantitative PCR (qPCR) analysis of ${ }^{\mathrm{MN} 1} \mathrm{CMP}-\mathrm{T} 21$ days after excision of exon 5 of Dot1l and found substantial downregulation of Hoxa9 (several 100-fold) (Figure 4C). Meis1, although also affected, was downregulated less consistently and to a lesser extent (0-5 fold, not statistically significant by qPCR). In order to evaluate gene expression changes on a whole transcriptome scale, we performed RNA-Seq of ${ }^{\mathrm{MN} 1} \mathrm{CMP}-\mathrm{T} 7$ days after introduction of Cre. Similar to the loss of Dot 1 in other model systems, a defined gene set was found to have decreased expression after loss of Dot1l (Figure 4D and Supplemental Table 3). The later Hoxa cluster genes (Hoxa7, Hoxa9, Hoxa10, and Hoxa11) were among the most dysregulated genes. The previously defined CMP/MN1 program showed significant enrichment in $\operatorname{Dot} 1 l^{f / f l}$ vs. Dot $1 l^{-/-}{ }^{\text {MN1 CMP-Ts, }}$ suggesting dependence of this program on functional DOT1L (Figure $4 \mathrm{E}$ ). The core enrichment of this signature (red shaded box in Figure 4E) defined a set of genes that are differentially expressed in MN1-leukemias (CMP/MN1 program) and dependent on functional DOT1L. The majority of these genes are associated with H3K79 methylation and bound by MN1 (Figure 4F). H3K79me2 ChIP-Seq tracks for the Hoxa cluster are shown in Figure 4G. 
A Chromosome 11

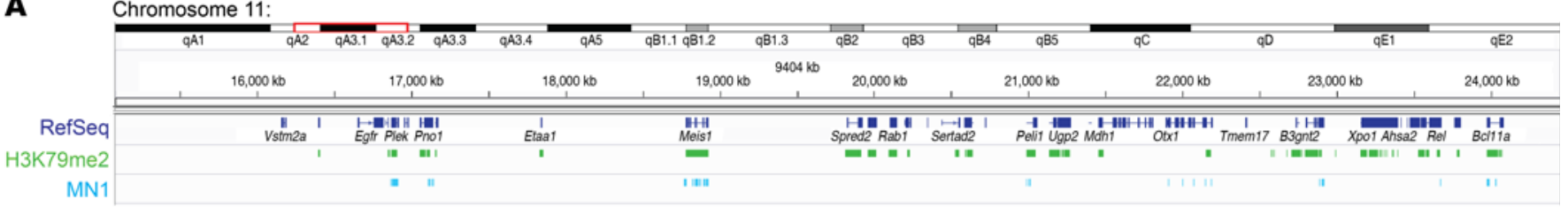

B

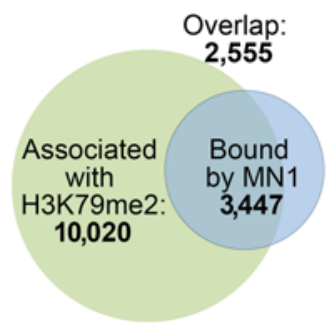

\section{Dot1 ${ }^{-{ }^{M N} 1}$ CMP-T}

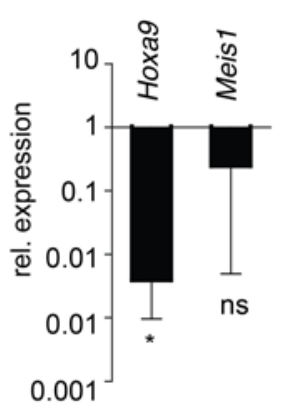

$\mathbf{F}$

F Core enrichment from $4 \mathrm{D}$ :

$$
\text { 80, overlap: } 46
$$

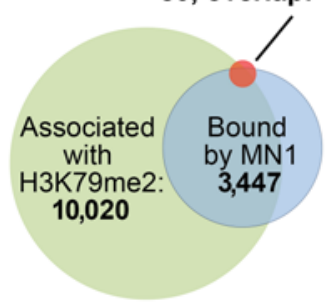

D Dot11 ${ }^{m}$ Dot11-

Top 30

Fam84a

Wt1

Evi1

Kynu

$A B C B 1$ a

Gria2

$D d \times 4$

$D d \times 3$

Hoxa10

Hoxa11

Glb1/2

Pde11a

Col4a1

Hoxa9

Hpgd

Hoxa7

Cd27

Marcks

II31ra

Myct1

Hlf

Filt3

Zfp503

Strp2

Epha3

Igf1

Tgm3

Hoxa5

Lypd6b

Egr1

51: Meis1 $(P<0.01)$

G

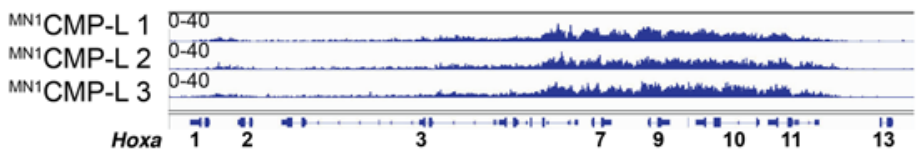

Figure 4. The MN1 cooperating program is downregulated after loss of Dot1l in MN1-transformed CMPs (MN1CMP-T). (A) ChIP-Seq for H3K79me2 in 3 MN1-driven murine leukemias. The IGV screen shot shows the overlap of genes associated with H3K79me2 with the genes bound by MN1 as assessed by ChIP-Seq by Heuser et al. (14). (B) Venn diagram showing overlap of genes associated with H3K79me2 (green) and bound by MN1 (blue). (C) qPCR for Hoxa9 and Meis1 in MN1CMP-T 21

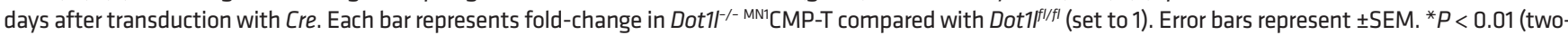

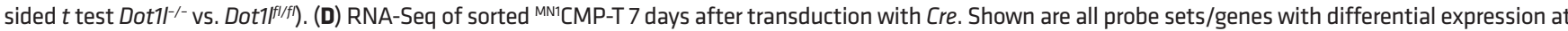
$P<0.01$, fold-change 2.5, as well as a list of the top 30 differentially expressed probe sets and Meis1. $n=3$ mice per group. (E) CSEA showing enrichment of the CMP/MN1 program in Dot 1/f/fl vs. Dot11/- MN1CMP-T. The core enrichment is marked by a red shaded box. Definition of core enrichment, NES, and $P$ value according to ref. 43. (F) Venn diagram of gene marked by H3K79me2 (green), bound by MN1 (blue), and the core enrichment of genes regulated by MN1 and dependent on DOT1L. (C) H3K79me2 methylation profile of the Hoxa cluster in MN1-driven CMP-derived leukemias ( $\left.{ }^{(M 1} \mathrm{CMP}-\mathrm{L}\right)$. Three individual leukemias are shown.

We also assessed enrichment of the genes dependent on DOT1L in ${ }^{\mathrm{MN}} \mathrm{CMP}-\mathrm{T}$ ("Dot1l-dependent in MN1") (Supplemental Table 2) in normal LSK vs. GMP, LSK Dot1 $1 t^{t / f l}$ vs. Dot $11^{-/}$, and MLL-AF9 leukemia Dot $1 l^{t / f l}$ vs. Dot $11^{-/}$data sets (Supplemental Figure 4, A-C); we

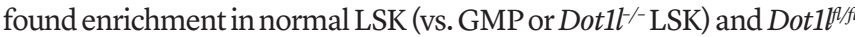
MLL-AF9 leukemias (vs. Dot1t/-). Finally, we asked whether the Dot1l-dependent in MN1 signature was also enriched in a second MN1-driven murine leukemia gene expression profile (16). Indeed, significant enrichment in MN1-transformed CMPs vs. normal CMPs was confirmed in this independent data set (Supplemental Figure 4D).

${ }^{M N 1}$ HSC-T growth and Hoxa9/Meis1 expression are independent of DOT1L in vitro. We isolated CMPs for transduction with MN1 in the experiments described above based on published results indicating transformed CMPs are the most efficient cell of origin in this model: MN1-transduced CMPs readily caused leukemia in recipient mice within 30-90 days, while MN1-transduced HSCs did not (14). Despite their inability to cause leukemia in mice, MN1-transduced HSC were able to serially replate. This was attributed to a lower expression of Hoxa9 in HSC-derived leukemias compared with CMPs - enough to allow in vitro immortalization but not enough to cause leukemia in an in vivo model (14). We asked whether DOT1L was also required for the (lower) Hoxa9 expression in MN1-transformed HSCs.

We isolated HSC-enriched populations from donor mice using two well-established flow cytometric approaches, LT-HSCs and LSK-SLAM. As previously reported, introduction of MN1 conferred serial replating potential to HSCs. We termed MN1-transduced 

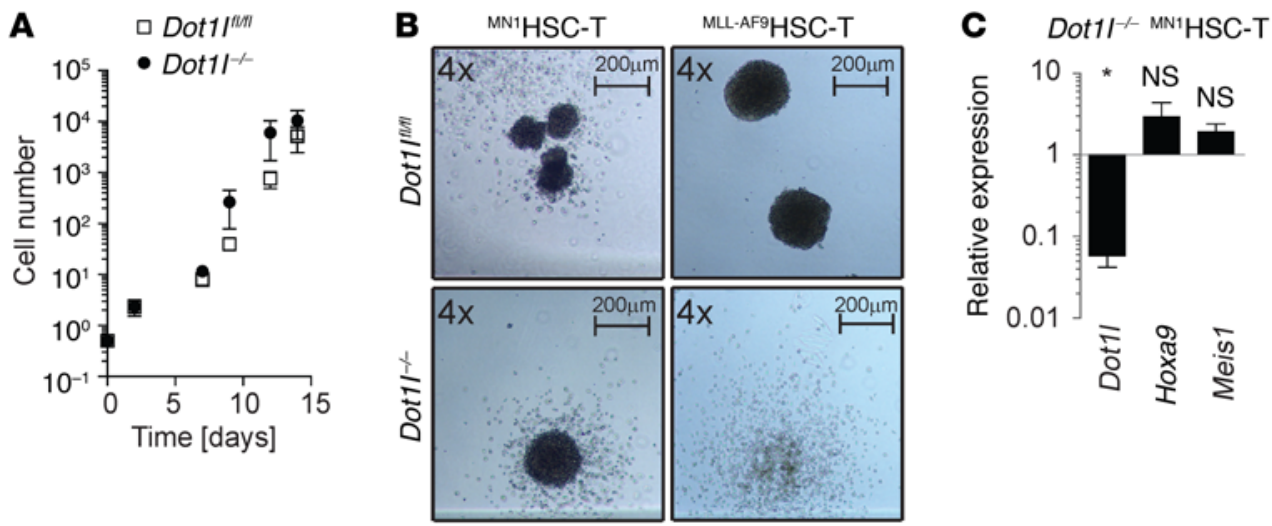

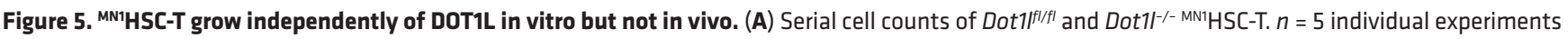

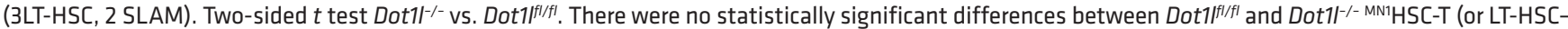

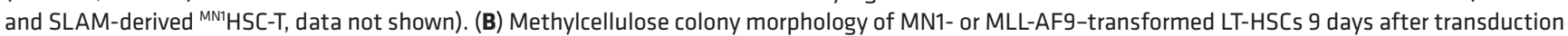

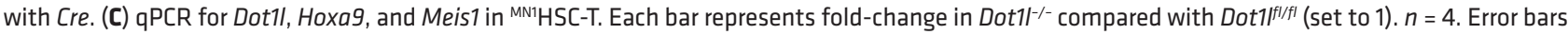

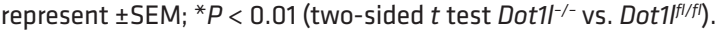

HSCs that were maintained in vitro ${ }^{\mathrm{MN}} \mathrm{HSC}-\mathrm{T}$ (transformed). To our surprise, ${ }^{\mathrm{MN} 1} \mathrm{HSC}-\mathrm{T}$ grew very well in vitro in complete absence of functional DOT1L (Figure 5A and Supplemental Figure 5, A-C), and Dot11 ${ }^{-{ }^{-M N}}{ }^{-} \mathrm{HSC}-\mathrm{T}$ were capable of forming blast-like colonies in methylcellulose (Figure 5B, left panel). In contrast to MN1-transformed LT-HSCs, MLL-AF9-transformed LT-HSCs required functional DOT1L. Dot11 ${ }^{-}{ }^{\text {MLLAF9 }} \mathrm{HSC}$-T formed less cellular and more dispersed colonies than Dot 1 llf $^{\text {Ifl MLL-AF9 }} \mathrm{HSC}-\mathrm{T}$, similar to what was previously observed with lineage-depleted or LSK-derived MLL-AF9 transformed cells (Figure 5B, right panels, and ref. 18). MLL-AF9-transformed LT-HSCs readily caused leukemia in mice, consistent with previously published results (27), and resulting leukemias were also DOT1L dependent (Supplemental Figure 5D).

We next investigated the transcriptional consequences of loss of Dot 1 lin ${ }^{\mathrm{MN} 1} \mathrm{HSC}$ - $\mathrm{T}$ and ${ }^{\mathrm{MN} 1} \mathrm{CMP}-\mathrm{T}$. MN1-transformed HSCs had previously been reported to express lower levels of Hoxa9 than MN1-transformed CMPs (14), and we confirmed this result (Supplemental Figure 5E, white bars). Of note, albeit lower, Hoxa9 was still clearly detectable in ${ }^{\mathrm{MN}}{ }^{\mathrm{H}} \mathrm{HC}$-T. Consistent with the lack of phenotypic changes, we did not find any statistically significant changes in Hoxa9 and Meis1 expression in ${ }^{\mathrm{MN} 1} \mathrm{HSC}-\mathrm{T}$ after inactivation of Dot1l (Figure 5C). DOT1L-independent Hoxa9 expression and growth were exclusively observed in vitro. Transplantation experiments confirmed that HSCs are inferior to CMPs as cell of origin for MN1-driven AML (Supplemental Figure 5F). Leukemias that did grow in vivo after injections of a large cell dose displayed Hoxa 9 expression levels and regulation similar to CMPderived leukemias (Supplemental Figure 5, G and H) and required functional DOT1L (Supplemental Figure 5, I and J). In addition, secondary transplants of ${ }^{\mathrm{MN} 1} \mathrm{HSC}-\mathrm{L}$ were also DOT1L dependent (Supplemental Figure 5, K and L) and revealed no substantial differences in penetrance, latency, phenotype, or clinical presentation to ${ }^{\mathrm{MN} 1} \mathrm{CMP}-\mathrm{L}$ (Supplemental Figure 5, M-P).

High HOXA9 expression is not universally associated with DOT1L dependence. The results from the ${ }^{\mathrm{MN}}{ }^{\mathrm{HSC}} \mathrm{H}-\mathrm{T}$ suggest that HOXA9 expression can be regulated in two distinct manners contingent on cellular or developmental context: DOT1L dependent and DOT1L independent. We asked whether DOT1L-independent HOXA9 expression is observed in human leukemias. To this end, we screened a small panel of leukemia cell lines for MN1 and HOXA9 expression (Figure 6, A and B). In this panel, we identified the Mutz3 cell line as having high $M N 1$ (consistent with previous reports; ref. 28) and high HOXA9 expression. In addition, high HOXA9 expression at a level comparable or greater than the $M L L$-rearranged control cell lines (MV4;11 and Molm14) was found in the early T cell precursor (ETP) ALL cell line Loucy, and in the AML cell line KG1 and its subline KG1a. We inhibited H3K79 methylation in KG1, KG1a, Loucy, and Mutz3 cells using the DOT1L inhibitor EPZ004777 (19). Despite high levels of HOXA9 expression (Figure 6A) and efficient decrease of the H3K79 methyl mark by Western blotting (Figure 6C), Loucy, KG1, and KG1a cells did not respond to DOT1L inhibition; phenotypically, we observed no change in growth (Figure 6, E and F), cell cycle, or apoptosis (Supplemental Figure 6, A and B). On a transcriptional level, there was no difference in HOXA9 expression with or without DOT1L inhibition (Supplemental Figure 6C). Despite a profound decrease in global H3K79 methylation, KG1 and KG1a retained some residual ChIP signal on the HOXA cluster (data not shown). We therefore cannot exclude that residual low-level H3K79me2 contributed to maintaining HOXA9 expression in these cells. However, ChIP-Seq over the HOXA cluster in Loucy cells demonstrated the complete absence of H3K79 methylation, yet HOXA9 expression was not affected (Figure 6D). HOXA9 expression is therefore independent of DOT1L in defined cellular contexts. In contrast, the $M N 1^{h /} H O X A 9^{h i} \mathrm{AML}$ cell line Mutz 3 was very sensitive to DOT1L inhibition, as demonstrated by decreased growth, increased apoptosis, and decreased cell cycle and HOXA9 expression (Figure 6, G-J).

Genetic inactivation of Mll1 impairs MN1-driven leukemogenesis. We next asked what could explain the DOT1L dependence of the MN1 leukemogenic program, since not all HOXA cluster expression appears to be DOT1L dependent. We had previously noted that WT MLL1 and DOT1L regulate very similar programs in LSK cells. We therefore asked whether MN1 cooperates with WT MLL1, 
A

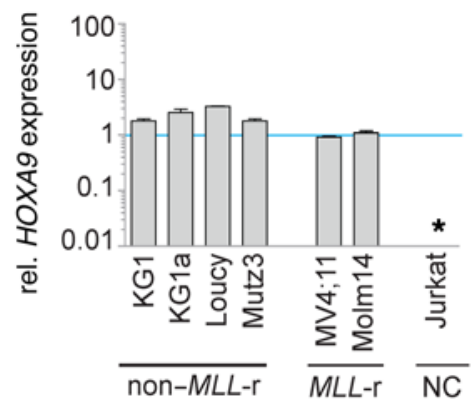

B

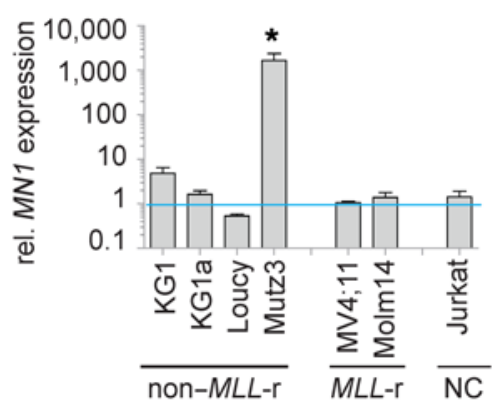

C

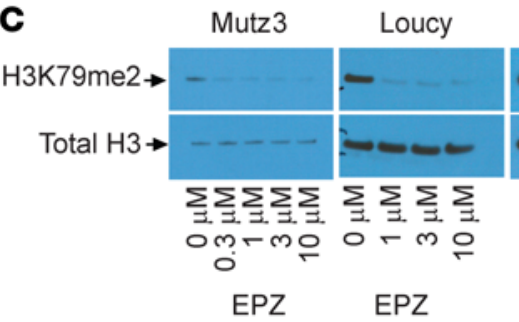

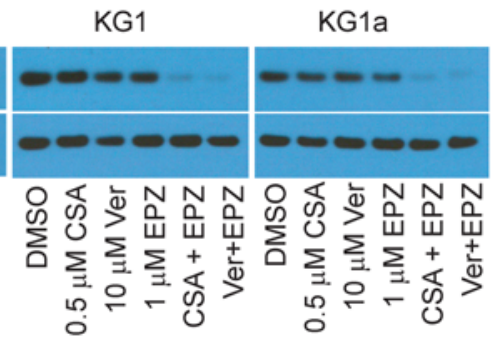

D

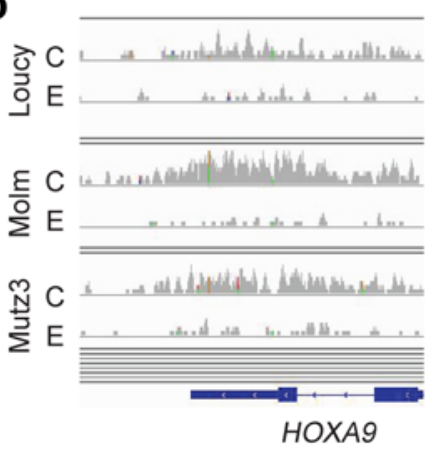

E

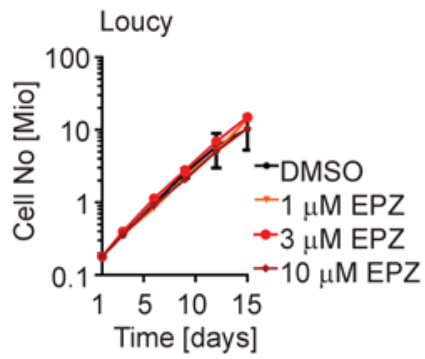

G

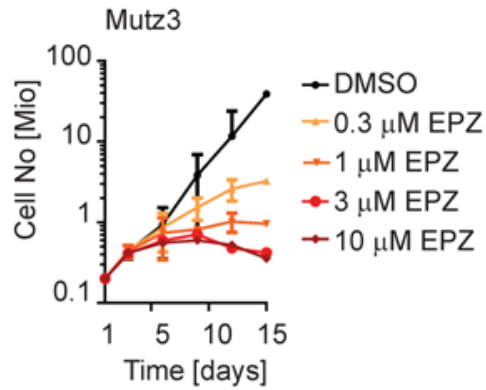

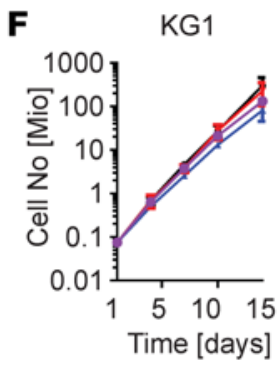

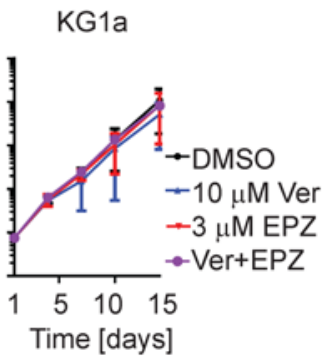

H

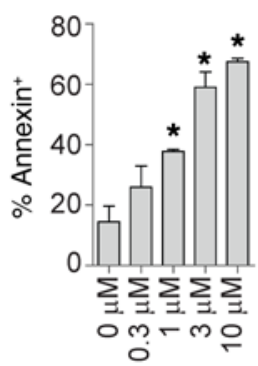

EPZ

I

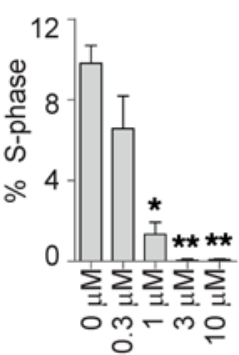

EPZ

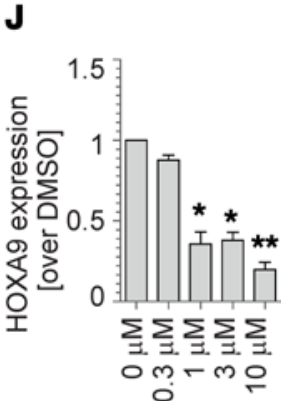

EPZ

Figure 6. $M N{ }^{\text {hi }} / \mathrm{HOXA}^{\text {hi }}$ Mutz3 cells respond to DOT1L inhibition while several other cell lines with high HOXA9 expression are unaffected. (A and B) qPCR for HOXA9 (A) and MN1 (B) in HOXA9 expressing non-MLL-rearranged (non-MLL-r) cell lines: Loucy (early T cell precursor ALL), KC1 (AML), KC1a (AML), and Mutz3 (AML). MLL-rearranged controls: MV4;11 (AML), Molm14 (AML). Negative control (NC): Jurkat. Each bar represents fold-change compared with Molm14 (set to 1); $n=3$. Two-sided $t$ test Dot11/- vs. Dot 1 $\left.\right|^{f / f f l}$. Error bars represent \pm SEM; ${ }^{*} P<0.01$ (ANOVA). (C) Western blot showing H3K79 dimethylation in Loucy, Mutz3, KG1, and KG1a cell lines exposed to the indicated concentration of the DOT1L inhibitor EPZ004777 (EPZ). KG1/KG1a cell lines exposed to the indicated concentration of the DOT1L inhibitor EPZ in the presence or absence of verapamil (Ver) or cyclosporine A (CSA). KG1 and KC1a express high levels of the efflux pump ABCB1/MDR1/P-GP, requiring blockade of the drug transported with verapamil or CSA to achieve efficient decrease in H3K79 methylation. (D) IGV tracks of Loucy, Mutz3, and Molm14 (Molm) treated with DMSO control (C) or $2 \mu$ M EPZ (E) over the HOXA9 locus confirm complete reduction of H3K79 dimethylation to background levels in treated cells. (E-C) Exposure of Loucy (E), KC1 and KC1a (F) to EPZ at the indicated concentrations. Shown are fold-expansion over a 14-day culture period (serial cell counts and Trypan Blue staining; $n=3$ independent experiments performed in duplicate; two-sided $t$ test; error bars represent \pm SEM). (G-J) Exposure of Mutz3 to EPZ at the indicated concentrations. Shown are fold-expansion over a 14-day culture period (serial cell counts and Trypan Blue staining) (G), apoptosis (Annexin staining) (H), cell cycle (\% cells in S-phase, EdU incorporation) (I), and HOXA9 expression (qPCR, fold change compared with DMSO set to 1$)(\mathrm{J}) . n=3$ independent experiments performed in duplicate. Two-sided $t$ test. Error bars represent \pm SEM; ${ }^{*} P<0.05$, ${ }^{* *} P<0.01$. 
A Colony number

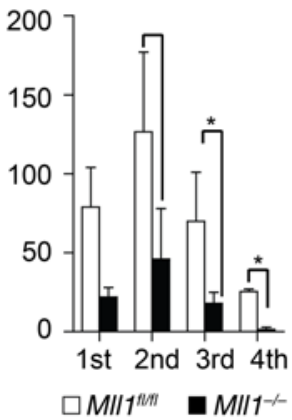

Cell number $[\mathrm{k} / \mathrm{plate}]$

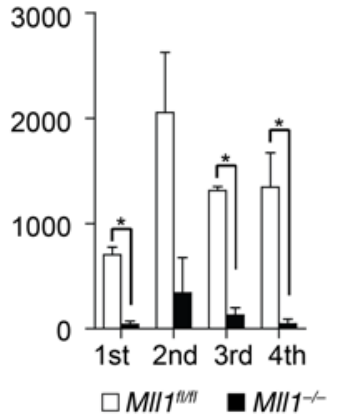

B
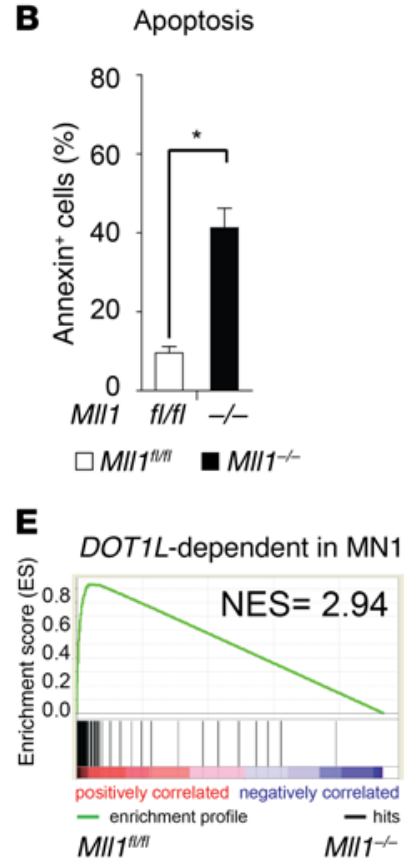

C

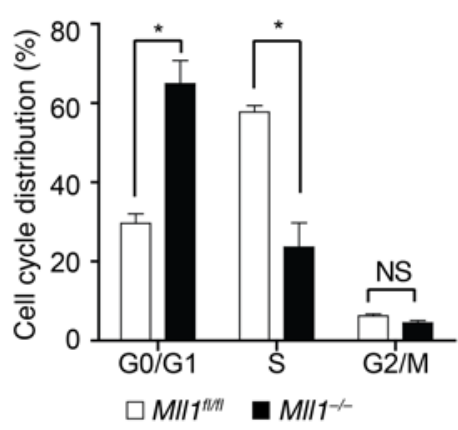

$\mathbf{F}$

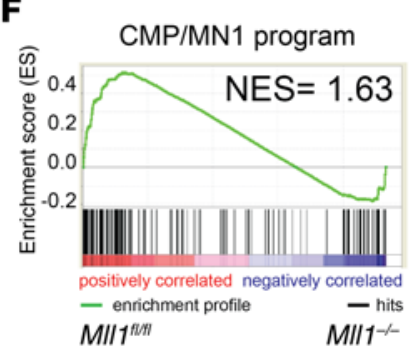

Figure 7. Loss of MII1 leads to decreased growth, increased apoptosis, and decreased in vivo leukemogenic activity of MN1-driven CMP-derived murine leukemia cells. (A) Serial replating of MN1-driven murine leukemias ( ${ }^{\mathrm{MN} 1} \mathrm{CMP}-\mathrm{L}$ ) after Cre-induced loss of MII1. Left plot: number of colonies per 500 plated cells; right plot: total cell number. Bulk population from 2 independent experiments; two-sided $t$ test in $M I / 1^{f l / f l}$ vs. $M I I 1^{-1 /}$. Error bars represent $\pm S E M$; ${ }^{*} P<$ 0.05. (B) Apoptosis (Annexin staining) in MN1-driven murine leukemias (MN1CMP-L) 1 week after deletion of MII1. Bulk population from 2 independent experi-

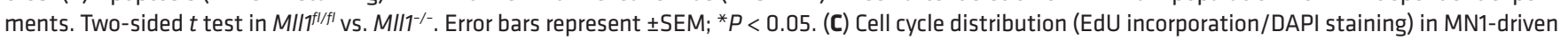

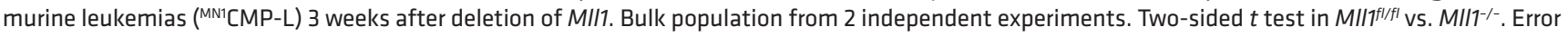
bars represent $\pm \mathrm{SEM}$; ${ }^{*} P<0.05$. (D) Survival of recipients of 100,000 MN1-driven CMP-derived leukemias (MN1CMP-L) transduced with Cre (MII1 ${ }^{-/-}$) or control

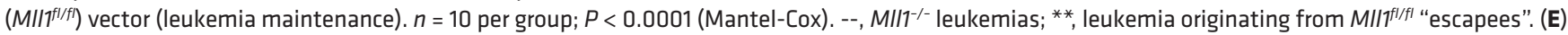

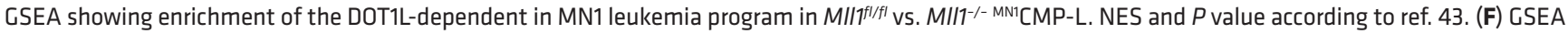

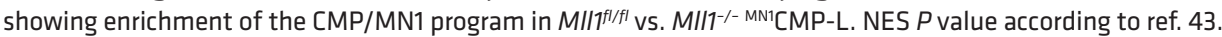

explaining the dependence on DOT1L. We transduced CMPs from Mll1 conditional KO mice (29) with MN1 and established Mll1 ${ }^{f / l} \mathrm{MN} 1$ leukemias. Similar to what we observed in the Dot $1 l$ conditional model, Cre-mediated inactivation of Mll1 resulted in decreased colony formation and cell growth, as well as increased apoptosis (Figure 7, A-C). Results from in vitro transformed cells also support a critical functional role for MLL1 (Supplemental Figure 7A). In vivo recipients of $M l l 1^{--}$MN1 leukemias had significantly prolonged survival compared with recipients of $\mathrm{Mll}^{\mathrm{f} / \mathrm{fl}}$ MN1 leukemias (Figure 7D and Supplemental Figure 7B). We next asked whether MLL1 and DOT1L regulated similar gene expression programs in MN1 leukemias. Indeed, the top 200 downregulated genes after genetic inactivation of Dot1l in MN1-transformed cells were strongly enriched in the genes downregulated upon genetic inactivation of Mll1 (Figure 7E and Supplemental Tables 2 and 3). Similarly, the most dysregulated genes in MN1-transduced CMPs compared with differentiation stage matched controls (Supplemental Table 1 in ref. 14) strongly enriched in $M_{l l 1} 1^{7 / f}$ versus Mll1 ${ }^{--}$MN1 leukemias (Figure 7F). The gene expression program that requires functional MLL1 in MN1 leukemias also substantially overlaps with the DOT1L-dependent program in MLL-AF9driven leukemias and in normal LSK cells (Supplemental Figure 7, C and D). Taken together, our data in the Mll1 conditional loss of function model suggest that MLL1 and MN1 coregulate a DOT$1 \mathrm{~L}$-dependent leukemogenic gene expression program.
High HOXA9 expression is observed in a subgroup of AML patient samples with high MN1 expression. Results from our Dot1l and Mll1 conditional mouse model and cell line data suggest that MN1-driven leukemias are dependent on high levels of HOXA9 expression, which in turn is regulated by MLL1 and DOT1L. This raises the possibility that targeting DOT1L could have therapeutic efficacy in $M N 1^{h i}$ AML. However, high MN1 expression in clinical AML is observed over a broad range of phenotypic, cytogenetic, and molecular subgroups, a heterogeneity that is not well captured in the retroviral MN1-overexpression mouse model or a single cell line. In order to investigate a potential role for DOT1L in clinical $M N 1^{h i}$ AML, we first asked whether HOXA9 and MEIS1 are coexpressed with MN1 in a substantial number of primary AML patient samples. We performed qPCR analysis of MN1, HOXA9, and MEIS1 from RNA of 25 primary AML patient samples (patient characteristics are summarized in Supplemental Table 4). MN1 is shown dichotomized at the median, the most commonly used cut-off to correlate MN1 with cytogenetics and outcome (Figure $8 \mathrm{~A}$, left axis, and refs. 1, 2, 5). Whenever possible, we confirmed MN1 expression levels with a second, standardized qPCR-based assay that reports ratios of MN1/ABL1 (Supplemental Figure 8A). We observed HOXA9 expression in 6 of 12 AML samples with high MN1 expression (Figure 8A, right axis). Elevated MEIS1 expression was observed in all HOXA9-expressing samples (Supplemental Figure 8, B and C). Moderately high MEIS1 expression was also 
A

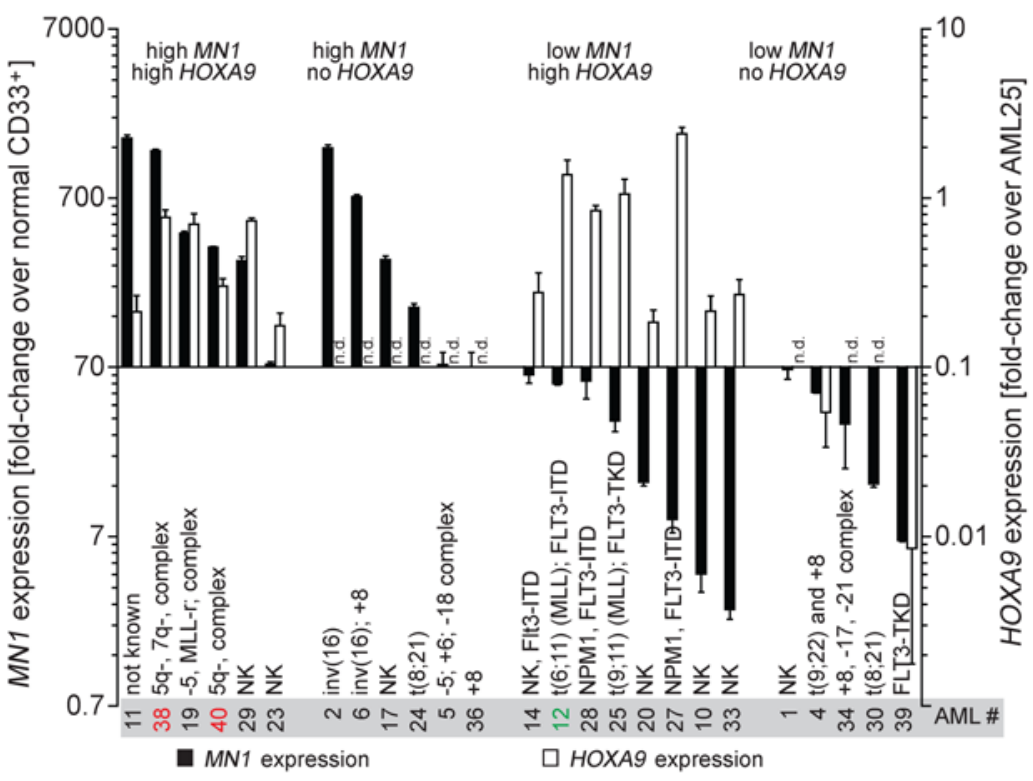

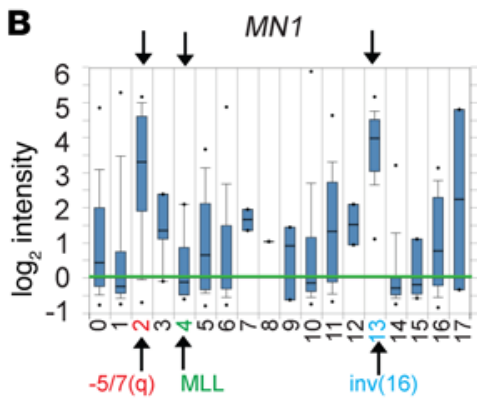

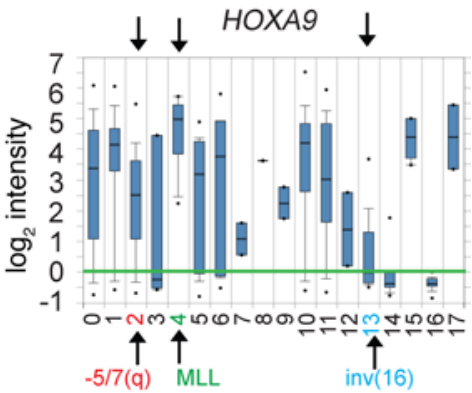

C

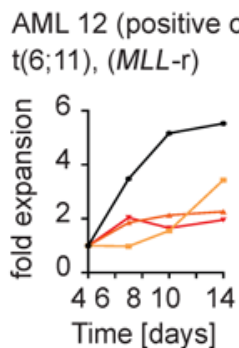

AML 38

5q-, 7q-, complex

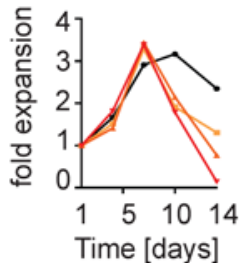

AML 40

5q-, complex

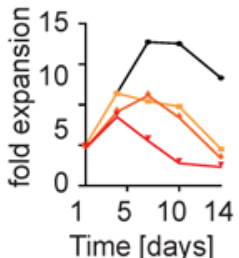

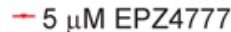

AML 51

$\operatorname{inv}(16)$

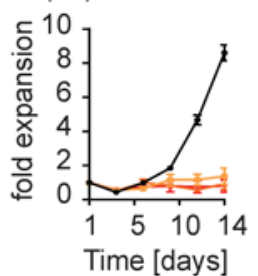

AML 24

$\mathrm{t}(8 ; 21),(A M L 1-E T O)$

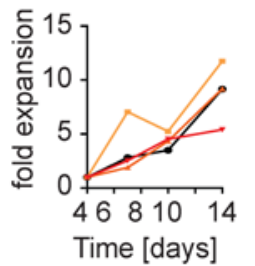

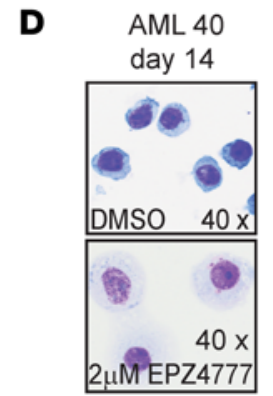
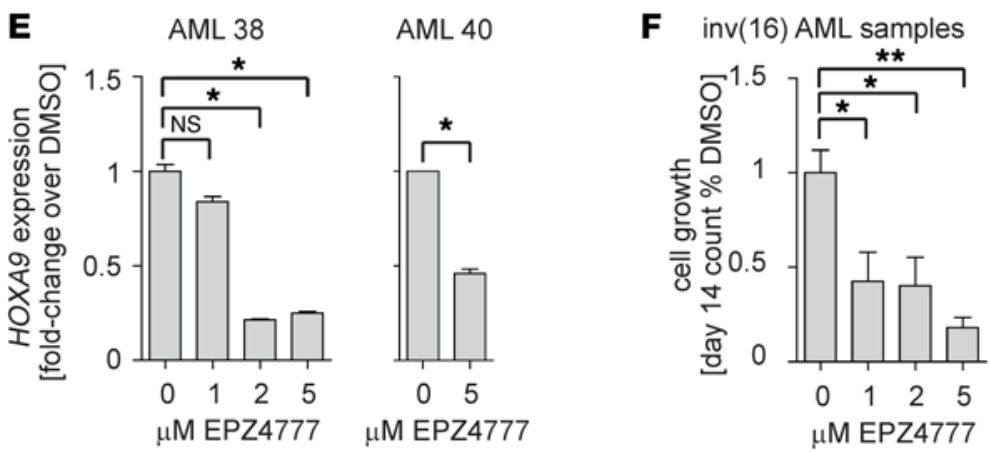

Figure 8. HOXA9 expression and sensitivity to DOT1L inhibition in MN1 ${ }^{\text {hi }}$ AML patient samples. (A) qPCR analysis of MN1 (left axis) and HOXA9 (right axis) in 25 primary patient AML samples. MN1 expression values are shown as fold-enrichment compared with normal CD33+ myeloid progenitors and plotted dichotomized at the median (median $=70$-fold overexpression). HOXA9 values are plotted as fold-enrichment compared with AML25 (MLL-rearranged, with known high HOXA9 expression set to 1). Error bars represent \pm SEM of 2-3 technical replicates. n.d., not detected. (B) MN1 and HOXA9 expression by genotype in Wouters Leukemia data set (Oncomine). Full legend: 0, not determined (90); 1, +8 (20); 2, -5/7(Q) (29); 3, -9q (6); 4, 11q23 (10); 5, complex (13); 6, failure (12); 7, MDS -7(Q) (2); 8, MDS -Y (1); 9, MDS complex (3); 10, normal (187); 11, other (53); 12, abn(3q) (2); 13, idv(16) (34); 14, $\mathrm{t}(15 ; 17)(21) ; 15, \mathrm{t}(6 ; 9)(6) ; 16, \mathrm{t}(8 ; 21)(35) ; 17, \mathrm{t}(9 ; 22)(2) . n=526$ AML samples. (C-F) Exposure of primary patient AML samples to the DOT1L inhibitor EPZ004777 at the indicated concentrations. AML12 (MLL-rearranged [MLL-r], positive control), AML38 (high MN1/HOXA9, complex karyotype with 5q-/7q-), AML 40 (high MN1/HOXA9, complex karyotype with 5q-), AML51 (inv[16], high MN1/no HOXA9), and AML24 (AML/ETO, intermediate high MN1/no HOXA9). Shown are fold-expansion over a 14-day culture period (serial cell counts and Trypan Blue staining; error bars represent duplicate counts) (C). Wright-Giemsa stain on cytospin of AML 40 (high MN1/HOXA9, complex karyotype with 5q-) treated with DMSO or EPZ004777 (D). HOXA9 expression in AML 38 and 40 (high MN1/HOXA9) treated with DMSO or EPZ004777. Error bars over technical replicates; ${ }^{*} P<0.05$ (twosided $t$ test) (E). Summary of cell growth of 3 inv(16) AML patient samples treated with DMSO or EPZ004777 (F). $n=3 ;{ }^{*} P<0.05$ (two-sided $t$ test). 
observed in several HOXA9- samples, including those with inv(16) (Supplemental Figure 8, B and C). Correlation with cytogenetics revealed that 3 samples with high $M N 1 / H O X A 9$ expression had a complex karyotype with loss of $5 q$ and/or 7q sequences (AML 38, 19 , and 40). On the other hand, we found that 6 of 12 AML samples with high MN1 expression had no detectable HOXA9/MEIS1 expression. The highest MN1 expression level in this group was observed in 2 samples with inv(16) (AML 2 and 6), which has previously been shown to be universally associated with MN1 overexpression $(4,6)$. Analysis of a well-annotated publicly available data set confirmed our results in a larger cohort of patients (30). The highest MN1 expression was found to be associated with two distinct cytogenetic subgroups, inv(16) and 5q-/7q-. As in our smaller cohort, HOXA9 was overexpressed in $5 \mathrm{q}-/ 7 \mathrm{q}-$, but not inv(16) AML (Figure 8B and Supplemental Figure 8D). AML with complex karyotype and 5q-/7q- often arises from myelodysplastic syndrome and is associated with poor outcome.

Two MN1 ${ }^{h i} /$ HOXA9 $^{\text {hi }}$ human AML samples are sensitive to DOT1L inhibition. We next asked whether $M N 1^{h i} H O X A 9^{h i}$ AML samples are sensitive to pharmacologic inhibition of DOT1L. Viably frozen cells were available from 2 patients (AML 38 and AML 40). Primary AML samples were maintained in culture on a feeder layer as recently described $(31,32)$ and exposed to the DOT1L inhibitor EPZ004777 in vitro (19). In AML 38 and 40, EPZ004777 induced a dose-dependent decrease in cell growth and in the fraction of cycling cells, as well as an increase in apoptosis (Figure 8C and Supplemental Figure $8 \mathrm{E}$ ). The observed effect was in a range comparable to AML 12 (MLL-rearranged). We also observed a dose-dependent upregulation of CD14, as well as a decrease in the nucleus/cytoplasma $(\mathrm{N}: \mathrm{C})$ ratio and increased vacuolization on cytospin consistent with differentiation (Figure 8D and Supplemental Figure 8E; differentiation was not assessed for sample AML 38). The observed phenotypic effects were associated with downregulation of HOXA9 (Figure 8E).

The very high MN1 levels in inv(16) AML also raised the questions of whether the cooperation between MN1 and DOT1L is more universal and whether DOT1L might play a role in this subtype. We therefore included 3 inv(16) primary patient samples in our analysis. Serial cell counts for AML 51 are shown in Figure 8C. Drug response for all 3 primary samples is summarized in Figure $8 \mathrm{~F}$ and suggests sensitivity to DOT1L inhibition in inv(16) AML. EPZ004777 administered together with verapamil to overcome MDR1-mediated exports also resulted in decreased growth and viability of the inv(16) cell line Me1 (Supplemental Figure 8, G and $\mathrm{H})$. The mechanism for the effect of EPZ004777 in inv(16) AML is almost certainly different, given the low levels of HOXA cluster expression typically observed in this subtype of AML. We performed transcriptome analysis of EPZ004777-treated and control inv(16) primary patient samples to delineate which gene sets are downregulated after DOT1L inhibition. We observed downregulation of gene sets associated with growth and cell cycle, consistent with the phenotypic results. Finally, we tested the susceptibility of AML 24 (AM1L/ETO) to DOT1L inhibition and found it to be unaffected (Figure 8C). This sample displayed moderately high MN1 expression - above the median but not as high as the inv(16) and 5q-/7q-/complex karyotype samples. Furthermore, this sample did not have any detectable expression of HOXA9.

\section{Discussion}

A previous study has identified a developmental gene expression program in CMPs that is downregulated at the transition to GMPs and is shared with MN1-driven murine leukemia. Key components of this program cooperate with forced expression of MN1 to cause AML (14). Our results suggest that expression of this program is regulated by two specific epigenetic modifiers, the histone methyltransferases MLL1 and DOT1L. CMP-derived MN1-transformed leukemias responded to conditional genetic inactivation of both $M l l 1$ and Dot $1 l$ with an increase in apoptosis and differentiation and a decrease in the fraction of cycling cells, as well as a decrease in ability to form leukemia in vivo. On a transcriptional level, loss of both MLL1 and DOT1L results in the downregulation of similar gene sets. These changes were associated with a collapse of the "MN1 susceptibility program," which included, as key players, the later HOXA cluster genes (HOXA7-13). Our data suggests a model wherein the aberrant expression of HOXA cluster genes (particularly HOXA9) beyond the normal developmental stage of a CMP can be caused by aberrant activation of MLL1 via translocation, partial tandem duplication, or overexpression of the transcriptional coregulator MN1. In each case, persistent expression of this program is dependent on DOT1L. A pharmacologic inhibitor of DOT1L is currently in clinical trials for leukemia carrying $M L L$-rearrangements ( $M L L$-translocations and partial tandem duplications [ref. 20], ClinicalTrials.gov, NCT02141828).

Interestingly, MN1-transduced HSCs gain serial replating capabilities in vitro but are inferior to CMPs in causing in vivo leukemia in recipient animals. This phenotype has been linked to a lower expression of Hoxa9 in MN1-transduced HSCs (14). It is in clear contrast to cell-of-origin requirements in the MLL-AF9driven mouse leukemia model; forced expression of MLL-AF9 induces high-level Hoxa9 expression and in vivo leukemogenic potential in cell-of-origin populations spanning the developmental stages of LT-HSC through GMPs, where the HOXA cluster is silenced $(27,33,34)$. It appears thus that MLL-AF9 is capable of directly increasing or reactivating the expression of later HOXA cluster genes in GMPs, while the transforming potential of MN1 is dependent on the endogenous expression levels of HOXA cluster genes in the cell of origin (14). The discrepancy in the efficiency with which MN1 and MLL-AF9 are able to cause full leukemic transformation in HSCs is probably more complex. We confirmed the lower Hoxa9 expression in HSC-derived MN1-transformed cells. Interestingly, there is no difference in Hoxa9 expression in normal HSCs and uncommitted progenitors (data not shown and ref. 35); thus, the failure of forced MN1 expression to cause leukemia has different causes in GMPs and HSCs.

Somewhat unexpectedly, we found Hoxa9 expression in HSCs immortalized by forced expression of MN1 to be independent of DOT1L. HOXA9 is overexpressed in about 50\% of AML (36), and most recent data has suggested that normal as well as malignant later HOXA cluster expression may require DOT1L independent of the expression of a leukemogenic fusion protein that could mediate direct recruitment $(23,37,38)$. We find that functional DOT1L is not universally required for high HOXA9 expression. The myeloid cell line KG1 and its subline KG1a, as well as the T-ALL cell line Loucy, all express HOXA9 at a level similar or 
higher to $M L L$-rearranged AML cell lines, yet HOXA9 expression - as well as cell growth and viability - are not affected by a substantial decrease (KG1, KG1a) or complete obliteration of H3K79 methylation globally and on the HOXA locus. Therefore, HOXA9 expression does not automatically imply DOT1L dependence. This underscores the need for in-depth mechanistic studies to identify subsets of AML that might be candidates for modulation of H3K79 methylation as a therapeutic approach.

The murine AML model caused by forced expression of MN1 in CMPs is consistently associated with overexpression of Hoxa9 and Meis1, which are critical for leukemogenesis. In contrast, human AML with high MN1 expression is considerably less uniform in patients. MN1 expression does not occur in two distinct clusters, but rather in a continuum, and is found across multiple phenotypic and cytogenetic subgroups. This poses considerable difficulties in defining a clinically meaningful cut-off, reflected in the inconsistencies of what constitutes high MN1 in several studies that investigated the prognostic impact of MN1 expression levels. We provide quantitative expression levels in a subset of samples in our cohort, which suggest that levels of $>10$ copies over ABL may be associated with biologically relevant $M N 1$ expression levels and DOT1L sensitivity. Similarly, expression levels at or above that of the Mutz3 cell line may constitute a good preliminary definition of high MN1. Confirmatory experiments in a larger cohort will be required to develop a precise and predictive clinical test for clinically relevant high $M N 1$ expression.

Analysis of publicly available AML data sets, as well as our own patient samples, suggests that about $50 \%$ of AML patients with high MN1 expression also express high levels of HOXA9. This constellation is enriched in AML with a complex karyotype and $5 q / 7 q$ abnormalities (30), an AML subtype with poor outcomes, and few defined therapeutic targets. Furthermore, expression of $M N 1$ and HOXA9 correlate with each other in patients with poor-risk cytogenetics (defined as complex karyotype/-5/-7), suggesting a mechanistic link in human AML (39). Our data strongly support further evaluation of DOT1L inhibition as a therapeutic strategy for this group of patients. Very high MN1 expression levels are also observed in inv(16) AML, which, in contrast to most other $M N 1^{\text {hi }}$ AML, is associated with a good prognosis $(4,6)$. We found sensitivity to DOT1L inhibition in one cell line and 3 patient samples with inv(16). HOXA9 expression is low or absent in this subtype, implying a mechanism of leukemogenesis that is not recapitulated in the MN1-CMP model (30). RNA-Seq analysis revealed modulation of gene sets associated with growth (E2F targets, Myc targets) and cell cycle (G2M check point, Cell Cycle CO) (Supplemental Table 8F). This is consistent with the observed phenotypic effects on growth and cell cycle. H3K79 methylation has been associated with cell cycle regulation in several experimental systems $(40,41)$. It is thus possible that the observed decrease in cell cycle regulatory programs is a direct consequence of the loss of $\mathrm{H} 3 \mathrm{~K} 79$ methylation. However, this would suggest that DOT1L inhibition should be more indiscriminately toxic to fast-cycling cells, which we and others found not to be the case. It therefore seems more likely that these changes reflect rather than cause the observed phenotypic effects. Future efforts will concentrate on expanding our findings in a larger cohort of samples and investigating possible mechanistic links between the CBFB-MYH11 translocation and DOT1L.
In summary, we found that deletion of either Mll1 or Dot1l profoundly compromises MN1-driven AML in a murine model; applicability of this finding to at least a subset of $M N 1^{h i}$ human AML is confirmed by the response of human AML with high MN1/HOXA9 expression in a cell line and patient samples to pharmacologic inhibition of DOT1L. These results suggest that DOT1L inhibition should be explored further in this poor prognostic subtype of AML.

\section{Methods}

For primer sequences, antibodies, and detailed experimental procedures, please refer to the Supplemental Methods.

Human samples. The samples from AML patients were obtained from diagnostic procedures at the University of Colorado Hospital (protocol 06-0720), with patient informed consent according to the Declaration of Helsinki and institutional review board approval from all participating centers.

qPCR analysis of human HOXA9 and MN1 in AML patient samples. Expression of $M N 1$ and HOXA9 was determined using Taq-man primer/ probes. Fold-change of $M N 1$ is shown compared with normal $C D 33^{+}$myeloid progenitors from two normal volunteers. HOXA9 is not expressed in normal CD33 $3^{+}$myeloid progenitors. Fold-change of HOXA9 was calculated compared with $M L L$-rearranged AML25. Absolute quantification of MN1 was performed using the MN1 Ipsogen kit (QIAGEN).

Dot1l and Mll1 KO mice, breeding. Animals were maintained at the Animal Research Facility at the University of Colorado Anschutz Medical Campus. Animal experiments were approved by the Internal Animal Care and Use Committee. Dot1l (18) and Mll1 (29) conditional KO mice were previously described and were maintained on a fully backcrossed C57BL/6 background.

Generation of transformed murine cells and leukemia. The MN1 cDNA was a gift from Ellen C. Zwarthoff (Erasmus University, Rotterdam, the Netherlands) and was cloned in the MSCV-IRES-GFP (MIG) plasmid. Ecotropic retroviral vectors containing murine MN1IRES-GFP, Cre-IRES-pTomato (Cre), and MSCV-IRES-pTomato (MIT) were generated by cotransfection of 293 cells. Lin-Sca- $1^{+} \mathrm{c}-\mathrm{Kit}^{+}$ $\mathrm{CD}_{4}^{-}{ }^{-} \mathrm{CD} 150^{+}$(SLAM), Lin ${ }^{-} \mathrm{Sca}-1^{+} \mathrm{c}-\mathrm{Kit}^{+} \mathrm{CD} 34^{-} \mathrm{Flk2} 2^{-}$(LT-HSC), Lin ${ }^{-}$ Sca-1 $1^{+} \mathrm{c}-\mathrm{Kit}^{+}$(LSK), or $\mathrm{Lin}^{-} \mathrm{Sca}-1^{-} \mathrm{c}-\mathrm{Kit}^{+} \mathrm{CD} 34^{+} \mathrm{Fc} \mathrm{R}^{\text {lo }}$ (CMP) cells were transduced with MN1-GFP and maintained with supplemental cytokines. After 2-7 days, $\mathrm{GFP}^{+}$cells were sorted and transduced with Cre or MIT. Two to 3 days after transduction, $\mathrm{GFP}^{+} / \mathrm{pTomato}^{+}$cells were sorted and transplanted into syngeneic irradiated recipients at $10^{5}$ cells/mouse. For secondary transplants, blast colonies were allowed to grow out from sorted $\mathrm{GFP}^{+} \mathrm{BM}$ cells. Leukemic cells were transduced with Cre or MIT, sorted, and transplanted.

Biochemical assays (apoptosis, cell cycle analysis, Western blotting, and $q P C R$ ). Cell growth and viability were followed by serial cell counts. Apoptosis and cell cycle analysis were performed using the Annexin-staining from BD Biosciences - Pharmingen and a Click-IT EdU kit (Molecular Probes, Invitrogen).

Cell growth assays for murine MN1 AML. For colony assays, sorted transduced leukemia cells were plated in methylcellulose M3234 containing IL3, IL6, and SCF at 1,000 cells per plate in duplicate and replated weekly at 500 cells/plate. Dot $1 l$ deletion was verified by PCR at each replating.

Cell growth assays and DOT1L inhibition for patient samples. Patient AML samples were maintained as described by Klco et al. 
(31). The DOT1L inhibitor EPZ004777 or DMSO control was added at the indicated concentrations. Cells were washed and replated in fresh compound every 3-4 days.

ChIP. ChIP for H3K79me2 in murine MN1 leukemias and human cell lines was performed using rabbit polyclonal antibodies from Abcam (catalog ab3594) similarly as described (42). ChIP DNA libraries were made following Illumina ChIP-seq library preparation kit and subjected to sequencing as below.

RNA amplification and gene expression array. RNA was isolated from sorted Dot1 $1 l^{\text {llfl }}$ or Dot $1 l^{-/}$LSK or ${ }^{\mathrm{MN} 1}$ CMP-T cells using Trizol (Invitrogen) or RNeasy mini columns (QIAGEN). RNA for array analysis was amplified, labeled, and hybridized to Affymetrix 430 2.A murine microarrays. RNA for RNA-Seq was submitted to the University of Colorado Denver genomics core for library preparation and sequencing.

Statistics. Array data was analyzed using GenePattern (http://www. broadinstitute.org/cancer/software/genepattern/). Raw sequences obtained from RNA-Seq were trimmed and mapped to $\mathrm{mm} 9$ using Short-read Nucleotide Alignment Program (GSNAP). Gene expression was calculated using CUFFLINKS (http://cole-trapnell-lab.github.io/ cufflinks/install/); differential gene expression was determined using ANOVA. GSEA was performed using www.broadinstitute.org/gsea. Shown are the normalized enrichment scores for the top gene sets enriched in DMSO-treated cells. Curated list of gene sets from MSigdb (Myc/E2F/proliferation associated datasets) were also used for GSEA. For genes associated with K79me2, ChIP-Seq, and Input reads were each combined into a single Bam file. MACS2 was used to identify peaks, which were intersected with mm9 RefSeq genes using Bedtools (intersectBed). Peaks were visualized using Integrated Genome Viewer (IGV) software. Venn diagrams were generated using BioVenn.

Gene expression and ChIP-Seq data was deposited at the NCBI Gene Expression Omnibus (GEO GSE54498, Expression changes after loss of Dot1l in murine LSK; GSE76750, ChIP-Seq and RNA-Seq).

Statistical analysis of colony and cell numbers, cell cycle, apoptosis, percent expression of markers associated with differentiation, and qPCR analysis was carried out using two-tailed Student's $t$ tests. Statistical analysis of survival was carried out using Kaplan Meyer estimates (Prism 5 software). A $P$ value of less than 0.05 was considered statistically significant.

Study approval. The murine studies were reviewed and approved by the University of Colorado Health Sciences Center Animal Care and Use Committee, University of Colorado Denver-Anschutz Campus, Aurora, Colorado, USA. The human studies were reviewed and approved by the Colorado Multiple Institutional Review Board (COMIRB), University of Colorado Denver - Anschutz Campus, Aurora, Colorado, USA. Samples were collected with patient informed consent prior to the procedure and in accordance with the Declaration of Helsinki.

\section{Author contributions}

SSR, KMB, and TN designed experiments. SSR, JNH, MB, BS, and $\mathrm{KMB}$ conducted experiments. DAP, QW, and CTJ provided patients samples, data, and technical help. PE provided $M l l 1^{f / f l}$ mice and technical help. AUS and TN performed biocomputational analysis. PE, DAP, SAA, and CTJ provided assistance in designing experiments and critically reviewed the manuscript. SSR, KMB, and TN wrote the manuscript. RMP and SRD provided EPZ004777 and assistance in designing experiments, confirmed DOT1L dependence of Mutz3 and MDR1-mediated drug efflux of EPZ004777 in independent experiments (data not included), and critically reviewed the manuscript.

\section{Acknowledgments}

We thank Ellen C. Zwarthoff (Erasmus University, Rotterdam, the Netherlands) for providing the MN1 plasmid. We thank Karen Helm and the staff at the flow cytometry core for assistance with cell sorting, and we thank Katrina Diener, Bifeng Gao, Ted Shade, and Kenneth Jones from the genomics and bioinformatics cores for assistance with the RNA-Seq experiment. This work was supported by start-up funds from University of Colorado Denver, Hematology/Oncology Section and the Children's Hospital Colorado Research Institute (to K.M. Bernt), as well as NHLBI K08HL102264 (to K.M. Bernt), Sidney-Kimmel Foundation 141761 (to K.M. Bernt), Doris-Duke Charitable Foundation 2014104 (to K.M. Bernt, for human cell line and patient sample work only; no funds from the Doris Duke Charitable Foundation were used to support research that used non-human animal models), NCI K08CA154777 (to T. Neff), ASCO career development grant (to D.A. Pollyea), and NCI P30CA046934 (Shared Resource of the University of Colorado Cancer Center).

Address correspondence to: Kathrin M. Bernt or Tobias Neff, Center for Cancer and Blood Disorders, Children's Hospital Colorado, University of Colorado, Denver, 13123 East 16th Ave., Box B115, Aurora, Colorado 80045, USA.Phone:303.724.7447; E-mail:Kathrin.Bernt@ ucdenver.edu (K.M. Bernt), Tobias.Neff@ucdenver.edu (T. Neff).

Amit U. Sinha's present address is: Basepair, New York, New York, USA.

Scott A. Armstrong's present address is: Department of Pediatrics, Human Oncology and Pathogenesis Program, and Leukemia Service, Department of Medicine, Memorial Sloan-Kettering Cancer Center, New York, New York, USA.
1. Heuser M, et al. High meningioma 1 (MN1) expression as a predictor for poor outcome in acute myeloid leukemia with normal cytogenetics. Blood. 2006;108(12):3898-3905.

2. Metzeler KH, et al. ERG expression is an independent prognostic factor and allows refined risk stratification in cytogenetically normal acute myeloid leukemia: a comprehensive analysis of ERG, MN1, and BAALC transcript levels using oligonucleotide microarrays. J Clin Oncol. 2009;27(30):5031-5038.
3. Langer C, et al. Prognostic importance of MN1 transcript levels, and biologic insights from MN1-associated gene and microRNA expression signatures in cytogenetically normal acute myeloid leukemia: a cancer and leukemia group B study. JClin Oncol. 2009;27(19):3198-3204.

4. Haferlach C, et al. Gene expression of BAALC, CDKN1B, ERG, and MN1 adds independent prognostic information to cytogenetics and molecular mutations in adult acute myeloid leukemia. Genes Chromosomes Cancer.
2012;51(3):257-265.

5. Xiang L, et al. The clinical characteristics and prognostic significance of MN1 gene and MN1-associated microRNA expression in adult patients with de novo acute myeloid leukemia. Ann Hematol. 2013;92(8):1063-1069.

6. Carella C, et al. MN1 overexpression is an important step in the development of inv(16) AML. Leukemia. 2007;21(8):1679-1690.

7. Lekanne Deprez RH, et al. Cloning and characterization of MN1, a gene from chromo- 
some 22q11, which is disrupted by a balanced translocation in a meningioma. Oncogene. 1995;10(8):1521-1528.

8. van Wely KH, et al. The MN1 oncoprotein synergizes with coactivators RAC 3 and $\mathrm{p} 300$ in RAR-RXR-mediated transcription. Oncogene. 2003;22(5):699-709.

9. Sutton AL, Zhang X, Ellison TI, Macdonald PN. The 1,25(OH)2D3-regulated transcription factor MN1 stimulates vitamin D receptor-mediated transcription and inhibits osteoblastic cell proliferation. Mol Endocrinol. 2005;19(9):2234-2244.

10. Buijs A, et al. Translocation (12;22) (p13;q11) in myeloproliferative disorders results in fusion of the ETS-like TEL gene on 12p13 to the MN1 gene on 22q11. Oncogene. 1995;10(8):1511-1519.

11. van Wely KH, Meester-Smoor MA, Janssen MJ, Aarnoudse AJ, Grosveld GC, Zwarthoff EC. The MN1-TEL myeloid leukemia-associated fusion protein has a dominant-negative effect on RAR-RXR-mediated transcription. Oncogene. 2007;26(39):5733-5740.

12. Grosveld GC. MN1, a novel player in human AML. Blood Cells Mol Dis. 2007;39(3):336-339.

13. Lai CK, et al. Cell fate decisions in malignant hematopoiesis: leukemia phenotype is determined by distinct functional domains of the MN1 oncogene. PLoS One. 2014;9(11):e112671.

14. Heuser M, et al. Cell of origin in AML: susceptibility to MN1-induced transformation is regulated by the MEIS1/AbdB-like HOX protein complex. Cancer Cell.2011;20(1):39-52.

15. Heuser M, et al. MN1 overexpression induces acute myeloid leukemia in mice and predicts ATRA resistance in patients with AML. Blood. 2007;110(5):1639-1647.

16. Kandilci A, Surtel J, Janke L, Neale G, Terranova S, Grosveld GC. Mapping of MN1 sequences necessary for myeloid transformation. PLoS One. 2013;8(4):e61706.

17. Imren S, et al. Modeling de novo leukemogenesis from human cord blood with MN1 and NUP98HOXD13. Blood. 2014;124(24):3608-3612.

18. Bernt KM, et al. MLL-rearranged leukemia is dependent on aberrant H3K79 methylation by DOT1L. Cancer Cell. 2011;20(1):66-78.

19. Daigle SR, et al. Selective killing of mixed lin- eage leukemia cells by a potent small-molecule DOT1L inhibitor. Cancer Cell. 2011;20(1):53-65.

20. Daigle SR, et al. Potent inhibition of DOT1L as treatment of MLL-fusion leukemia. Blood. 2013;122(6):1017-1025.

21. Jones B, et al. The histone H3K79 methyltransferase Dot1L is essential for mammalian development and heterochromatin structure. PLoS Genet. 2008;4(9):e1000190.

22. Chen L, et al. Abrogation of MLL-AF10 and CALM-AF10-mediated transformation through genetic inactivation or pharmacological inhibition of the H3K79 methyltransferase Dot1l. Leukemia. 2013;27(4):813-822.

23. Deshpande AJ, et al. AF10 regulates progressive H3K79 methylation and HOX gene expression in diverse AML subtypes. Cancer Cell. 2014;26(6):896-908.

24. Jo SY, Granowicz EM, Maillard I, Thomas D, Hess JL. Requirement for Dot1l in murine postnatal hematopoiesis and leukemogenesis by MLL translocation. Blood. 2011;117(18):4759-4768.

25. Nguyen AT, He J, Taranova O, Zhang Y. Essential role of DOT1L in maintaining normal adult hematopoiesis. Cell Res. 2011;21(9):1370-1373.

26. Essers MA, et al. IFN $\alpha$ activates dormant haematopoietic stem cells in vivo. Nature. 2009;458(7240):904-908.

27. Krivtsov AV, et al. Cell of origin determines clinically relevant subtypes of MLL-rearranged AML. Leukemia. 2013;27(4):852-860.

28. Valle VD, Guglielmi L, Busson M, Zwarthoff EC, Berger R, Bernard OA. Expression of the MN1-TEL fusion protein in the human UCSD/AML1 leukemic cell line. Leukemia. 2004;18(9):1558-1560.

29. Artinger EL, et al. An MLL-dependent network sustains hematopoiesis. Proc Natl Acad Sci U S A. 2013;110(29):12000-12005.

30. Wouters BJ, Lowenberg B, Erpelinck-Verschueren CA, van Putten WL, Valk PJ, Delwel R. Double CEBPA mutations, but not single CEBPA mutations, define a subgroup of acute myeloid leukemia with a distinctive gene expression profile that is uniquely associated with a favorable outcome. Blood. 2009;113(13):3088-3091.

31. Klco JM, et al. Genomic impact of transient lowdose decitabine treatment on primary AML cells.
Blood. 2013;121(9):1633-1643.

32. Sarkaria SM, Christopher MJ, Klco JM, Ley TJ. Primary acute myeloid leukemia cells with IDH1 or IDH2 mutations respond to a DOT1L inhibitor in vitro. Leukemia. 2014;28(12):2403-2406.

33. Krivtsov AV, et al. Transformation from committed progenitor to leukaemia stem cell initiated by MLL-AF9. Nature. 2006;442(7104):818-822.

34. Cozzio A, Passegue E, Ayton PM, Karsunky H, Cleary ML, Weissman IL. Similar MLL-associated leukemias arising from self-renewing stem cells and short-lived myeloid progenitors. Genes Dev. 2003;17(24):3029-3035

35. Cabezas-Wallscheid N, et al. Identification of regulatory networks in HSCs and their immediate progeny via integrated proteome, transcriptome, and DNA methylome analysis. Cell Stem Cell. 2014;15(4):507-522.

36. Argiropoulos B, Humphries RK. Hox genes in hematopoiesis and leukemogenesis. Oncogene. 2007;26(47):6766-6776.

37. Chang MJ, et al. Histone H3 lysine 79 methyltransferase Dot1 is required for immortalization by MLL oncogenes. Cancer Res. 2010;70(24):10234-10242.

38. Deshpande AJ, et al. Leukemic transformation by the MLL-AF6 fusion oncogene requires the H3K79 methyltransferase Dot1l. Blood. 2013;121(13):2533-2541.

39. Heuser M, et al. Modeling the functional heterogeneity of leukemia stem cells: role of STAT5 in leukemia stem cell self-renewal. Blood. 2009;114(19):3983-3993.

40. Kim W, Choi M, Kim JE. The histone methyltransferase Dot1/DOT1L as a critical regulator of the cell cycle. Cell Cycle. 2014;13(5):726-738.

41. Schulze JM, et al. Linking cell cycle to histone modifications: SBF and $\mathrm{H} 2 \mathrm{~B}$ monoubiquitination machinery and cell-cycle regulation of $\mathrm{H} 3 \mathrm{~K} 79$ dimethylation. Mol Cell. 2009;35(5):626-641.

42. Krivtsov AV, et al. H3K79 methylation profiles define murine and human MLL-AF4 leukemias. Cancer Cell. 2008;14(5):355-368.

43. Subramanian A, et al. Gene set enrichment analysis: a knowledge-based approach for interpreting genome-wide expression profiles. Proc Natl Acad Sci U S A. 2005;102(43):15545-15550. 